\title{
Quantum Computing and Cognitive Simulation
}

\author{
Martha Lewis* \\ Department of Engineering Mathematics, University of Bristol
}

\section{Introduction}

Cognitive science is the study of the mind and how it relates to human behaviour. As a field, it is highly interdisciplinary, bringing together theories from psychology, neuroscience, biology, and others. The field of cognitive science operates at a number of levels. On the one hand, some aspects of cognitive science look at the behaviour of neurons, whereas other aspects look to explain human behaviour at a more abstract level, seeking to explain human decision making or reasoning. There is a long-standing history of vector space models in cognitive science. Theories of categorization such as those developed by Ashby and Gott (1988); Nosofsky (1986); Rosch (1975) utilise notions of distance and similarity that can readily be incorporated in vector space models of meaning. Hampton (1987); Smith and Osherson (1984); Tversky (1977) encode meanings as feature vectors, and models of high-level cognitive reasoning have been implemented within vector symbolic architectures (Smolensky, 1990; Gayler, 2003; Plate, 1995).

Another field in which vector space models play an important role is physics, and especially quantum theory. Though seemingly unrelated to language, intriguing connections have recently been uncovered. The link between physics and natural language semantics that vector space models provide has been successfully exploited, providing novel solutions and a fresh perspective for a number of problems related to cognitive science, such as modelling logical aspects in vector spaces (Widdows and Peters, 2003). Methods from quantum logic have also been applied to cognitive processes related to the human mental lexicon, such as word association (Bruza et al., 2009), decision-making (Pothos and Busemeyer, 2013), and human probability judgements (Busemeyer et al., 2011). Furthermore, the categorical model of Coecke et al. (2010), inspired by quantum mechanics, has provided a convincing account of compositionality in vector space models and an extensible framework for linguistically motivated research on sentential semantics. More recently, the link between physics and text meaning was made more concrete by a number of proposals that aim at replacing the traditional notion of a word vector with that of a density matrix - a concept borrowed from quantum mechanics which can be seen as a probability distribution over vectors (Piedeleu et al., 2015; Bankova et al., 2019; Sadrzadeh et al., 2018)

A key area of research in cognitive science is how to formalise human behaviours around judgements of similarity, categorization, and decision making. However, formalising concepts within a vector space, and relying on distance within vector space to give a notion of similarity can lead to some puzzling features, at least if we assume that distance and similarity behave classically. For example, it has been shown (Tversky, 1977) that judgements of similarity are not always symmetric, as you would expect if they are just based on distance in a vector space.

In the field of physics, quantum mechanics has fundamentally altered our understanding of the way in which particles behave. Quantum mechanics has a number of unintuitive phenomena, some of which can be used model unusual aspects of human behaviour. This is not to say that a description of the brain and mind is provided in terms of quantum phenomena at the small scale, although this sort of modelling has been proposed (Penrose, 1990; Hameroff, 2014). Instead, the idea is that the formalism arising from quantum theory provides the right sort of mathematical tools to model these puzzling aspects of human behaviour.

* To appear in the book Quantum Computing in the Arts and Humanities: An Introduction to Core Concepts, Theory and Applications. E. R. Miranda (Ed.). Cham: Springer Nature, 202x. Pre-publication draft v.27 Mar 2021. 
The application of quantum theory to model human behaviour is wide-ranging. In this chapter we will look at three main areas in which it has been applied. One key area is in how similarity judgements can be modelled. There are a number of phenomena around similarity judgements that are not well modelled using a view of concepts that does not take the state of the observer into account. These include the asymmetry of similarity judgements and the fact that similarity can change depending on other exemplars that are present (Tversky, 1977). These phenomena have been addressed in Pothos et al. (2013). Another key area is in judgement and decision-making. Again, puzzling phenomena have been observed regarding the fact that judgements do not follow classical probability theory, known as the conjunction fallacy (Tversky and Kahneman, 1983), can depend crucially on the order in which questions are presented (Moore, 2002) or on the amount of knowledge about the world (Tversky and Shafir, 1992). These phenomena are modelled within a quantum framework in Franco (2009); Busemeyer et al. (2011); Wang and Busemeyer (2013); Pothos and Busemeyer (2009); Khrennikov and Haven (2009). Related to this area is the phenomenon of contextuality. This can be summarised as the idea that there may be sets of random variables (in psychology, results of an experiment) that have pairwise joint distributions but for which no joint distribution across the whole set of random variables can be found. This is one of the key aspects of quantum theory. Amazingly, the same theory was also developed in psychology, and has been formalised in Dzhafarov and Kujala (2014, 2016). Finally, quantum theory has been applied to describe categorisation and concept combination. Again, the ways in which humans use concepts has been shown not to be well modelled by classical views of combination like fuzzy set theory, where problems known as over- and under-extension are observed. (Smith and Osherson, 1984; Hampton, 1987, 1988a,b, 1997). Approaches to answer these phenomena have been proposed in Aerts and Gabora (2005a,b); Aerts (2009); Sozzo (2014, 2015); Aerts et al. (2015). The problem of modelling how concepts compose can also be addressed via a linguistic route. The problem of modelling concept composition by means of the grammatical structure in language has been addressed in Coecke et al. (2010), and applications of this to the problem of overextension was proposed in Coecke and Lewis (2015).

Approaches to modelling cognitive phenomena at the neural level have also been considered within a quantum-theoretic framework. Fuss and Navarro (2013) show that a quantum random walk approach to modelling choice tasks better simulates human reaction times. Consideration of neuronal activation withing a quantum framework is also discussed in Pothos and Trueblood (2015). The question of modelling how concepts can 'bind' together (for example, the combination of an adjective and a noun) was addressed at a vector-based level in Smolensky (1990) and has been investigated within a cognitive science context in Martin and Doumas (2020). The compositional distributional framework of Coecke et al. (2010) has the potential to model these aspects of neuronal activity well, and combining this theory with the tensor product binding of Smolensky (1990) is an area for future research.

In the remainder of the chapter we cover the following. We provide a short section on the mathematical notation we will use. In section 2 we will summarize the cognitive phenomena that have been described by quantum theory. In section 3 we describe how quantum theory has been used to address each of these phenomena, as well as discussing quantum-theoretic approaches to modelling neuronal-level phenomena. In section 4 we discuss the implications for using quantum computing to model cognitive phenomena and artificial intelligence more generally.

\subsection{Mathematical notation}

We assume that the reader has a general understanding of linear algebra. We use Dirac's bra-ket notation to represent vectors, their duals, and inner products.

- A ket $|v\rangle$ is a column vector in a Hilbert space $\mathcal{H}$. We will always consider the space to be finitedimensional, so $\mathcal{H}=\mathbb{C}^{n}$

- A bra $\langle v|$ is the vector dual to $|v\rangle$. It is the conjugate transpose of $|v\rangle$ and can be thought of as a row vector whose elements are the complex conjugates of the elements in $|v\rangle$

- The inner product of two vectors $|v\rangle,|w\rangle$ is represented by $\langle v \mid w\rangle$. 
- If a matrix is represented by $M$, its multiplication with a vector $|v\rangle$ is given by $M|v\rangle$ and its multiplication with a bra $\langle v|$ is given by $\langle v| M$. Often these will be combined to give a scalar $\langle v|M| v\rangle$.

- The absolute value of a complex number $\alpha=a+i b$ is represented as $|\alpha|=\sqrt{a^{2}+b^{2}}$

- The Euclidean norm of a vector is written $\|v\|=\sqrt{\langle v \mid v\rangle}$

\section{Cognitive phenomena}

One of the cornerstones of human cognition is the ability to categorize. From an evolutionary perspective it is essential to be able to categorize objects into edible/inedible, or animals into dangerous/safe. Central to the notion of categorization is the notion of similarity. An animal can be classified as safe based on how similar it is to another animal known to be safe. The study of categorization and similarity is therefore central to cognitive science, and has been widely researched (Ashby and Gott, 1988; Nosofsky, 1986; Rosch, 1975). How can we determine whether one item is similar to another or not? One of the approaches to studying the notion of similarity and categorization is to view objects as represented by points in a feature space, and then determining similarity within that space. Categories can then be viewed as forming regions within the feature space. However, under classical assumptions this kind of model does not necessarily model human behaviour well. We describe here some of these key phenomena.

\subsection{Asymmetry of similarity judgements}

In Tversky (1977) the question is addressed of whether objects can be represented as points in a geometric space together with distance between the points being measured via a metric on the space. Similarity between objects is then viewed as a function of the distance between the objects in the space.

Within this model, a key assumption is that distance is viewed as a metric, meaning that the following hold: given objects $x, y$, and $z$, and distance metric $d$,

$$
\begin{aligned}
& d(x, x)=0 \\
& d(x, y)=d(y, x) \text { (symmetry) } \\
& d(x, z) \leq d(x, y)+d(y, z) \text { (triangle inequality) }
\end{aligned}
$$

Tversky (1977) show that these assumptions do not necessarily hold. In a series of experiments, Tversky showed that when one object is considered more prominent than another, the less prominent object is considered more similar to the more prominent object that vice-versa. Firstly, pairs of countries are assessed (by Israeli college students) for which country is most prominent. The pairs of countries are, for example, China and Vietnam ${ }^{1}$, USA and Mexico, or Belgium and Luxembourg. A separate group of students were then asked to judge which phrase they preferred to use when describing the similarity of the two countries out of "country $a$ is similar to country $b$ " and "country $b$ is similar to country $a$ ". Across all pairs, the majority of students chose the ordering in which the more prominent country was given second, for example "Vietnam is similar to China" rather than "China is similar to Vietnam". This kind of effect was seen across a range of topics and modalities, for example when judging similarity of shapes, letters, and sequences of sounds.

\subsection{Diagnosticity}

Tversky (1977) further show that judgements of similarity can be altered by the presence of comparison objects. According to a geometric model of similarity, the similarity of two objects should not be affected by the presence of other objects. However, this is shown not to hold for human similarity judgements in the following manner. Pairs of quadruples of countries were designed that differ in only one entry, i.e. there are pairs of sets $\{a, b, c, p\}$ and $\{a, b, c, q\}$. Participants are asked to say which of $b, c$ and $q$ or $p$ country $a$

\footnotetext{
${ }^{1}$ In fact, the countries assessed are Red China and North Vietnam
} 
is most similar to. For example, one such pair is the sets of countries \{Austria, Sweden, Poland, Hungary and A Austria, Sweden, Norway, Hungary\}. Participants see only one quadruple and are asked to judge which country Austria is most similar to. Tversky (1977) find that in the set \{Austria, Sweden, Poland, Hungary\}, most participants judge Austria to be most similar to Sweden, but in the quadruple \{Austria, Sweden, Norway, Hungary\} participants judge Austria to be most similar to Hungary.

\subsection{Conjunction and disjunction fallacies}

In modelling human decision making, one assumption is that human behaviour can be expressed by means of probabilities. A key facet of classical probability is that the probability of a conjunction of events will always be less than or equal to the probability of one of its constituents. $P(A \& B) \leq P(A)$. Similarly, the probability of a disjunction of events will always be greater than or equal to the probability of one of its constituents: $P(A) \leq P(A$ or $B)$. However, experiments in Tversky and Kahneman (1983) showed that these rules are not adhered to when humans make decisions about category membership. The experiments run as follows. Human participants are given a story about a person, and asked to make certain judgements about them. The most frequently cited is the following:

Linda is 31 years old, single, outspoken and very bright. She majored in philosophy. As a student, she was deeply concerned with issues of discrimination and social justice, and also participated in anti-nuclear demonstrations.

The description is followed by eight statements about the person's career or personality:

- Linda is a teacher in elementary school.

- Linda works in a bookstore and takes Yoga classes.

- Linda is active in the feminist movement. $(F)$

- Linda is a psychiatric social worker.

- Linda is a member of the League of Women Voters.

- Linda is a bank teller. $(T)$

- Linda is an insurance salesperson.

- Linda is a bank teller and is active in the feminist movement. (T\&F)

Participants are asked to rank the eight statements associated with each description by how probable each statement is. A large majority (between 85 and 90\%, depending on the participant pool) rank the statements in the order $T \& F>T$, that is, they judge that the probability that Linda is both a feminist and a bank teller is higher than the probability that Linda is a bank teller. This phenomenon still holds (to a lesser extent) in other contexts where the participants are asked to place a bet on the two statements $T$ and $T \& F$, in a domain of medical experts (where the story that is told is about symptoms and the task is to diagnose the patient), and in a range of other situations designed to reduce the incidence of the conjunction fallacy. Experiments where the fallacy does not hold to such a great extent are when the participant pool is with a range of statistically sophisticated participants, and when the experiment is phrased in terms of numbers of people. 


\subsection{Question order models}

In Moore (2002) the effect of order on answers to questions is addressed. If you are asked 'Do you try to eat healthily?' and 'Do you like McDonalds?', your answer to each question may change depending on which question is asked first. There are a number of ways in which the ordering could affect the answers. Moore identifies four types of such effect, termed contrast, consistency, additive, and subtractive. The consistency effect runs as follows. A group of participants is asked a pair of questions. For a concrete example consider the questions "Do you think Bill Clinton is trustworthy" and "Do you think Al Gore is trustworthy". For context, these questions were asked in 1997. If the question is asked first, it is considered to be asked in a non-comparative context, meaning that there is nothing to immediately compare the question to. If the question is asked second, it is considered to be asked in a comparative context, because it can be considered in comparison to the first. The answers to the questions were distributed as in table 1 . The effect of the question ordering is that the answer to the question in the comparative context is altered to make it more consistent with the answer in the non-comparative context. So, if a participant is first asked whether Gore is honest and trustworthy, and they answer positively, then they are more likely to answer positively to the question of whether Clinton is honest and trustworthy. On the other hand, if a participant is first asked whether Clinton is honest and trustworthy, and answers negatively, then they are more likely to answer negatively to the question of whether Gore is honest and trustworthy. The effect is to make the answers to the two questions more consistent.

\begin{tabular}{l|c|c|c} 
& \multicolumn{3}{|c}{ Percent saying yes } \\
Context & Clinton & Gore & Gap \\
\hline First & 50 & 68 & $+18^{*}$ \\
Second & 57 & 60 & +3 \\
Difference & $+7^{*}$ & $-8^{*}$ & $-15^{*}$ \\
\hline
\end{tabular}

Table 1: Example of the consistency effect. ${ }^{*}$ indicates significant difference. Figures from Moore (2002)

The contrast effect occurs when people alter their answers to make the answers contrast with each other. For example, in a Gallup poll in 1995 when asked the question of whether the label "honest and trustworthy" applies to either Bob Dole or Newt Gingrich, people's answers in the comparative context altered to increase the gap between them, emphasizing the differences between the two. Figures are shown in table 2

\begin{tabular}{l|c|c|c} 
& \multicolumn{4}{|c}{ Percent saying yes } \\
Context & Gingrich & Dole & Gap \\
\hline First & 41 & 60 & $+19^{*}$ \\
Second & 33 & 64 & $+31^{*}$ \\
Difference & $-8^{*}$ & $+4^{*}$ & $+12^{*}$ \\
\hline
\end{tabular}

Table 2: Example of the contrast effect. ${ }^{*}$ indicates significant difference. Figures from Moore (2002)

Under the additive effect, considering either question first has the same effect. In a series of racial hostility polls in 1996, people were asked the questions "Do you think that only a few white people dislike blacks, many white people dislike blacks, or almost all white people dislike blacks?" and "Do you think that only a few black people dislike whites, many black people dislike whites, or almost all black people dislike whites". In both cases, the percentage responding "All" or "Many" increased in the comparative context, see table 3

Lastly, in the subtractive context considering either question first again has the same effect, but in this case the effect is to decrease the number of positive answers. The example given in Moore (2002) is the question of whether baseball players Pete Rose and, separately, Shoeless Joe Jackson should be eligible for ad- mission to the Baseball Hall of Fame. In each case, the players are ineligible, and the participants are told why. In each case, the number of people answering favourably to the question of whether they should be allowed decreased in the comparative context (table 4) 


\begin{tabular}{l|c|c|c} 
& \multicolumn{3}{|c}{ Percent saying "All" or "Many" } \\
Context & White & Black & Gap \\
\hline First & 41 & 46 & +5 \\
Second & 53 & 56 & +3 \\
Difference & $+12^{*}$ & $+10^{*}$ & -2 \\
\hline
\end{tabular}

Table 3: Example of the additive effect. ${ }^{*}$ indicates significant difference. Figures from Moore (2002)

\begin{tabular}{l|c|c|c} 
& \multicolumn{3}{|c}{ Percent saying "Favourable" } \\
Context & Rose & Jackson & Gap \\
\hline First & 64 & 45 & $+19^{*}$ \\
Second & 52 & 33 & $+19^{*}$ \\
Difference & $-12^{*}$ & $-12^{*}$ & 0 \\
\hline
\end{tabular}

Table 4: Example of the subtractive effect. ${ }^{*}$ indicates significant difference. Figures from Moore (2002)

These four examples show clearly the different kinds of effects that question order can have on responses, and that the context in which a question is asked is crucial for its response. These effects are addressed in Wang and Busemeyer (2013), Wang et al. (2013)

\subsection{The 'sure thing' principle}

A counter-intuitive aspect of human decision making is the violation of the 'sure thing' principle. The sure thing principle was introduced by Savage (1954). This says that if we prefer $x$ to $y$ given any possible state of the world, then we should prefer $x$ to $y$ even when the exact state of the world is unknown. However, people do not always make decisions according to the sure thing principle. Tversky and Shafir (1992) present the following experiment:

Imagine that you have just taken a tough qualifying examination. It is the end of the fall quarter, you feel tired and run-down, and you are not sure that you passed the exam. In case you failed you have to take the exam again in a couple of months - after the Christmas holidays. You now have an opportunity to buy a very attractive 5-day Christmas vacation package to Hawaii at an exceptionally low price. The special offer expires tomorrow, while the exam grade will not be available until the following day. Would you:

1. buy the vacation package

2. not buy the vacation package

3. pay a $\$ 5$ non-refundable fee in order to retain the rights to buy the vacation package at the same exceptional prices the day after tomorrow - after you find out whether or not you have passed the exam.

$31 \%$ of respondents said that they would buy the vacation package, $7 \%$ said that they would not buy the vacation package, and $60 \%$ said that they would reserve the low price for the small fee - paying to wait until the uncertainty was resolved.

However, when the participants were asked to imagine they they knew they outcome of the exam, a majority said that they would buy the package:

Imagine that you have just taken a tough qualifying examination. It is the end of the semester, you feel tired and run-down, and you find out that you [passed the exam/failed the exam. You will have to take it again in a couple of months - after the Christmas holidays]. You now have an opportunity to buy a very attractive 5-day Christmas vacation package to Hawaii at an exceptionally low price. The special offer expires tomorrow. Would you 
1. buy the vacation package

2. not buy the vacation package

3. pay a $\$ 5$ non-refundable fee in order to retain the rights to buy the vacation package at the same exceptional prices the day after tomorrow.

In the context where the participant had passed the exam, $54 \%$ of respondents said that they would buy the vacation package, $16 \%$ of respondents said that they would not by the vacation package, and $30 \%$ of student said that they would pay the $\$ 5$ waiting fee. Very similarly, in the case where the participant had failed the exam, $57 \%$ of respondents said that they would buy the vacation package, $12 \%$ of respondents said that they would not by the vacation package, and $31 \%$ of student said that they would pay the $\$ 5$ waiting fee. Essentially, in the context where the outcome of the exam is known, a majority of participants decide to book the holiday - whether or not it is pass or fail. According to the sure thing principle, those participants should decide to book the holiday in that case where the outcome of the exam is not known. But, as can be seen, this does not happen.

A similar experiment involves gambling:

Imagine that you have just played a game of chance that gave you a $50 \%$ chance to win $\$ 200$ and a $50 \%$ chance to lose $\$ 100$. The coin was tossed and you have [won $\$ 200 /$ lost $\$ 100$ ].

You are now offered a second identical gamble

$50 \%$ chance to win $\$ 200$ and $50 \%$ chance to lose $\$ 100$. Would you:

1. Accept the second gamble

2. Reject the second gamble

In the context that the participants had won the first gamble, $69 \%$ accepted and $31 \%$ rejected the second. In the context that participants had lost the first gamble, the split was $59 \%$ accept, $41 \%$ reject. Either way, a majority of participants accepted the second gamble.

However, in a context where they did not know the outcome of the first gamble, a majority of the same set of participants rejected the second gamble. The split was $36 \%$ accept, $64 \%$ reject.

The explanation given in Tversky and Shafir (1992) is that the presence of uncertainty makes it more difficult for people to focus on the implications of each outcome.

A further example is given in a prisoner's dilemma situation. In the prisoner's dilemma, a hypothetical prisoner has the choice either to cooperate with the other prisoner, i.e. keep quiet about their activities, or defect, i.e. tell the authorities what they know. The possible outcomes of cooperating or defecting are presented in the following payoff matrix:

\begin{tabular}{c|cc} 
& Other D & Other C \\
\hline You D & You 10, Other 10 & You 25, Other 5 \\
You C & You 5, Other 25 & You 20, Other 20
\end{tabular}

Table 5: Matrix of payoffs for the prisoner's dilemma situation

In an experimental situation, if the participant is told that the other has cooperated, the majority of participants choose to defect. Also, if the participant is told that the other has defected, the majority of participants choose to defect. However, if the participant is not told what the choice of the other is, the majority of participants choose to cooperate.

\subsection{Categorization}

One of the most important things that humans can do is to categorize their experiences. Is this fruit good to eat, or poisonous? Is that large striped animal dangerous? Categorization can be thought of in terms of similarity to other experiences, and to help us do this we use concepts. There have been a number of 
theories of concepts. The classical view of concepts dates back to Plato and Aristotle. Under the classical view of concepts, a concept is viewed as a set of necessary and sufficient conditions, formulated as a list of properties. Such a view of concepts is inadequate. Firstly, for many natural concepts it is impossible to give a set of necessary and sufficient conditions Wittgenstein (1953). Instead, instances of a category can be thought of as sharing a set of 'family resemblances'. For example: what makes the images in figure 1 pictures of chairs, and the images in figure 2 not pictures of chairs? It is difficult to decide on a set of necessary and sufficient conditions that rules the kneeling chair to be a chair, but rules out a bar stool.

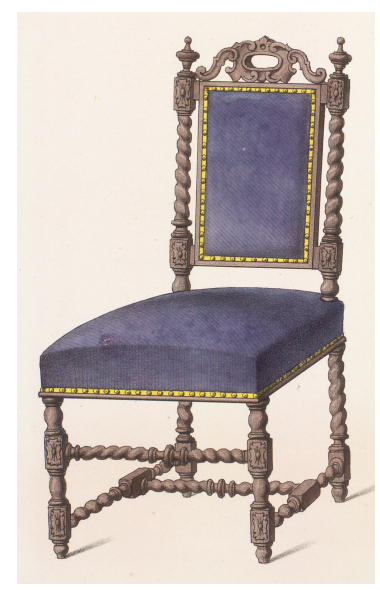

(a) Upholstered Chair ${ }^{2}$

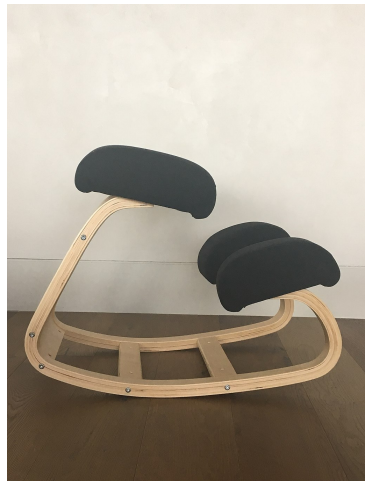

(b) Kneeling Chair ${ }^{3}$

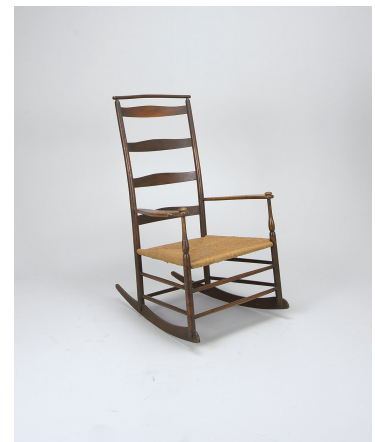

(c) Rocking Chair ${ }^{4}$

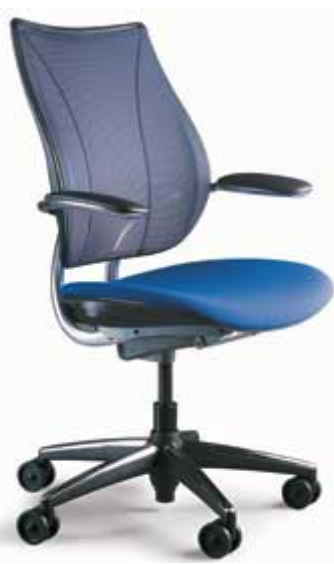

(d) Office Chair ${ }^{5}$

Figure 1: Images of chairs.

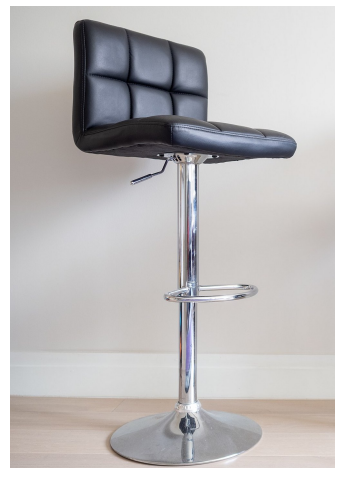

(a) Bar Stool $^{6}$

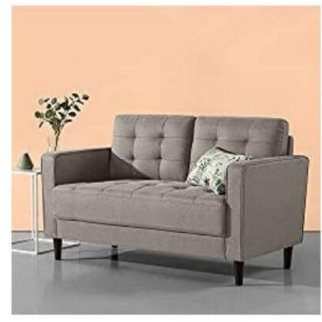

(b) Sofa $^{7}$

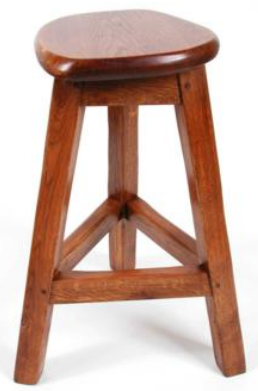

(c) $\mathrm{Stool}^{8}$

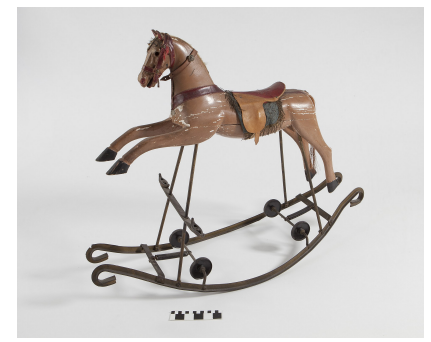

(d) Rocking Horse ${ }^{9}$

Figure 2: Non-chairs.

Furthermore, the classical view does not take into account the graded and context-dependent nature of concepts. Consider the much simpler concept tall. Whether or not to describe something as tall will firstly

\footnotetext{
${ }^{2}$ https://commons.wikimedia.org/wiki/File:Philipp_Rumpf_Studienblatt_eines_Stuhls.jpg

${ }^{3}$ https://commons.wikimedia.org/wiki/File:Sleekform_Kneeling_Chair.jpg

${ }^{4}$ https://commons.wikimedia.org/wiki/File:Shaker_no._7_rocking_chair_Rocking_Chair,_1878\%E2\%80\%931910_(CH_ 18460985).jpg

${ }^{5}$ https://commons.wikimedia.org/wiki/File:LibertyChair.jpg

${ }^{6}$ https://commons.wikimedia.org/wiki/File:Bar_Stools_(49907001456).jpg

${ }^{7}$ https://commons.wikimedia.org/wiki/File:52.8_Inch_Sofa_Couch_Loveseat,_Grey.jpg

${ }^{8}$ https://commons.wikimedia.org/wiki/File:TabouretAFDB.jpg

${ }^{9}$ https://commons.wikimedia.org/wiki/File:Wooden_Rocking_Horse_with_Weels.jpg
} 
be dependent on the set of things that form its context, for example 4 year old children, or Dutch women, or giraffes, or mountains. Secondly, even if we have fixed a context, it is not a crisp question whether we can divide some people as tall. In figure 3, how are we to decide what is the necessary and sufficient condition for being tall? Certainly anyone to the right of Jeremy Clarkson is tall, and anyone to the left of Nikita Khrushchev is not tall, but should we state the condition to be $180 \mathrm{~cm}$ ? $184 \mathrm{~cm}$ ? If we say $184 \mathrm{~cm}$ and someone is $183.5 \mathrm{~cm}$, are they really not tall?

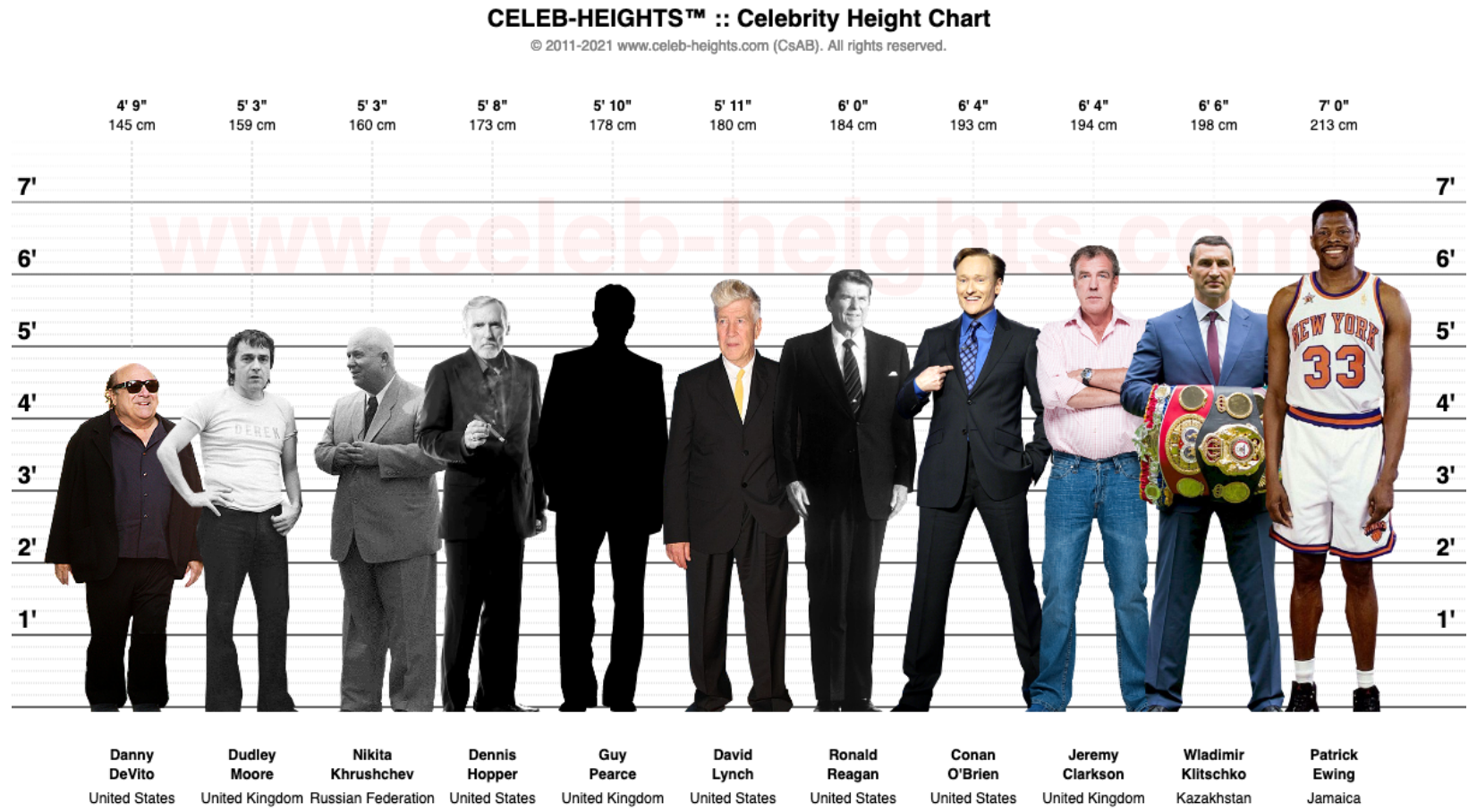

Figure 3: Heights of male celebrities

These sorts of consideration led to the formulation of prototype theory (Rosch, 1975). In prototype theory, concepts are assumed to have a prototype, and members of the concept are judged as being more or less similar to the prototype. For example, in the concept bird, a robin is judged as a very prototypical bird, whereas an ostrich or a penguin would be judged as much less typical. This allows the consideration of concepts in terms of family resemblances, and accounts for the graded nature of concepts.

Further, the exemplar theory of concepts views a category as based on specific instances, or exemplars, that are stored in memory. Categorization judgements are based on judgements of similarity to the exemplars, which are easily called to mind.

Exemplar theory and prototype theory are both backed by experimental evidence (Rosch, 1975; Nosofsky, 1986). Key characteristics of these theories are that they can account for the graded nature of concepts, and in both cases, we represent concept instances within some kind of feature space, equipped with a distance metric.

Another key feature of human concept use is that we are able to combine concepts to create new ones. This can be done in very simple ways, for example combining the concept blue and the concept book, we 


\begin{tabular}{c|cccccccc}
\multirow{2}{*}{ Suspect } & \multicolumn{2}{|c}{ tall } & \multicolumn{2}{c}{ not tall } & \multicolumn{2}{c}{ tall and not tall } & \multicolumn{2}{c}{ neither tall nor not tall } \\
& True & False & True & False & True & False & True & False \\
\hline $5^{\prime} 4^{\prime \prime}$ & 1.3 & 98.7 & 94.7 & 3.9 & 14.5 & 76.3 & 27.6 & 65.8 \\
$5^{\prime} 7^{\prime \prime}$ & 5.3 & 93.4 & 78.9 & 17.1 & 21.1 & 65.8 & 31.6 & 57.9 \\
$5^{\prime} 11^{\prime \prime}$ & 46.1 & 44.7 & 25 & 67.1 & 44.7 & 40.8 & 53.9 & 42.1 \\
$6^{\prime} 2^{\prime \prime}$ & 80.3 & 10.5 & 9.2 & 82.9 & 28.9 & 56.6 & 36.9 & 55.3 \\
$6^{\prime} 6 "$ & 98.7 & 1.3 & 0 & 100 & 5.3 & 81.6 & 6.6 & 89.5
\end{tabular}

obtain a blue book. How can we formalize this? One approach to formalizing concept combination used fuzzy set theory. Under this view, a concept is a fuzzy set, that is, a set whose members has membership values in the interval $[0,1]$ rather than in the set $\{0,1\}$. We write

$$
\mu_{A}: X \rightarrow[0,1]
$$

and use the notation $\mu_{A}(x)$ to describe the membership of the item $x$ in the concept $A$ In fuzzy set theory we can form the conjunction or disjunction of two sets as follows:

$$
\begin{aligned}
\mu_{A \text { and } B}(x) & =\min \left(\mu_{A}(x), \mu_{B}(x)\right. \\
\mu_{A \text { or } B}(x) & =\max \left(\mu_{A}(x), \mu_{B}(x)\right)
\end{aligned}
$$

Under these rules, combinations quickly become problematic. The classic example is that of a pet fish Smith and Osherson (1984). Consider a fish like a goldfish. A goldfish is not a particularly typical fish, nor a very typical pet, so we might assign low memberships to goldfish in each of these concepts:

$$
\begin{array}{r}
\mu_{\text {pet }}(\text { goldfish })=0.3 \\
\mu_{\text {fish }}(\text { goldfish })=0.25
\end{array}
$$

However, it is a very typical pet fish, i.e. we would assign something like

$$
\mu_{\text {pet fish }}(\text { goldfish })=0.9
$$

The question then is what is going on in the combination of these concepts to allow the typicality of the instance in the combined concept to be higher than it is in either of the individual concepts.

This kind of phenomenon was examined in Hampton $(1988 \mathrm{a}, \mathrm{b})$. In the 'pet fish' kind of example, where typicality of an item in a conjunction of concepts is higher that the typicality of an item in one or more of its constituent concepts, the phenomenon is called 'overextension'. On the other hand, in the case of disjunction of concepts, a phenomenon termed underextension is also seen. In this case, typicality of an item in the disjunction of two concepts is lower than typicality in one or more of the constituent concepts.

\subsection{Negation}

Another problematic phenomenon in human concept use is the use of negation. In Alxatib and Pelletier (2011), an experiment is performed where the membership of humans in the category 'tall' is measured. Imagine we have five suspects in a robbery, all men, with heights $5^{\prime} 4^{\prime \prime}, 5^{\prime} 7^{\prime \prime}, 5^{\prime} 11^{\prime \prime}, 6^{\prime} 2^{\prime \prime}, 6^{\prime} 6^{\prime \prime}$. Participants are asked to judge, of each suspect, whether they are a) tall, b) not tall, c) tall and not tall, or d) neither tall nor not tall. The percentages of people agreeing to these statements are given in the table below. Note that in the experiment, the suspects were shown in a randomized order; we present them here in height order for ease of presentation.

There is a strong correlation between the values for the borderline statements "tall and not tall" and "neither tall nor not tall". Participants were willing to agree to both of these statements. Int the first statement "tall and not tall", a participant would seem to ascribe the value true both to "tall" and to "not tall", whereas in the second, the participant would also judge that he is neither. 
The problem of negation is also considered by Hampton, in a setting combined with conjunction Hampton (1997). In this setting, Hampton considers the typicality of items to conjunctions of concepts. For example, we can consider the typicality of parrot to the conjunction Pets which are also birds and Pets which are not birds. Of course, we would consider the typicality of parrot in the first to be high and in the second to be low. However, if concepts are being combined in line with logical reasoning, then the sum of the typicality in Pets which are also birds and in Pets which are not birds should not exceed the typicality of parrot in Pets. This is another form of overextension, as we have seen in previous descriptions.

\section{Quantum models}

The problems for modelling cognitive phenomena outlined in section 2 above have all been tackled using methods from quantum theory. Below, in section 3.1 we give an overview of the key ideas before explaining the models in detail. After this overview, we discuss approaches to modelling similarity, diagnosticity, and the conjunction fallacy. These approaches have been developed over a research programme laid out in Busemeyer et al. (2011); Pothos et al. (2013); Franco (2009) and other papers, and crucially depend on the notion of interference. Following this, we consider the sure thing principle and question order models. Again, these models depend on the notion of interference. We go on to discuss models of concept combination and negation. These approaches use the quantum phenomenon of entanglement as well as interference to describe how humans combine concepts. Linked to these is the notion of contextuality. Finally, we talk about quantum models that are applied at a neuronal level.

\subsection{Overview of quantum models in psychology}

The following gives an overview of some of the key quantum concepts used in approaches such as those in Pothos et al. (2013); Busemeyer et al. (2011). Within applications of quantum theory to psychology, questions are considered from the point of view of an agent. WE consider an agent as having a certain state $|p s i\rangle$, and as questions are asked of the agent, or the agent is exposed to a stimulus, the state of the agent changes. Within quantum theory, a question about a system is termed an observable: a self-adjoint linear operator. The eigenvectors $\left\{\left|a_{i}\right\rangle\right\}_{i}$ of an observable $A$ form an orthonormal basis of the Hilbert space, and the eigenvalues $\left\{a_{i}\right\}$ associated with the $\left\{\left|a_{i}\right\rangle\right\}_{i}$ form the values that the observable can take, i.e. the answers to the questions. To give a simple example: a yes/no question such as 'is the sky blue'? might be encoded in a two-dimensional vector space. An observable $B$ on the space will have two orthonormal eigenvectors $|b l u e\rangle$ and $\mid$ not blue $\rangle$. We can set the eigenvalues associated with $|b l u e\rangle$ and $\mid$ not blue $\rangle$ to be 1 and 0 respectively.

Now, we represent an agent's belief about the state of the sky state of the sky as a vector $|s\rangle$. Given that the agent is ambivalent about what the weather is (possibly clear and blue, possibly overcast and grey), the probability that we receive the answer 'yes!' to the question 'Is the sky blue' is given by the following calculation:

$$
P(\text { yes })=|\langle b l u e \mid s\rangle|^{2}
$$

This quantity is the square of the projection onto the blue axis, illustrated in figure 4

This notion can be extended. Firstly, there may be more than one basis vector corresponding to a given answer, so that that answer corresponds to a subspace of the vector space of dimension greater than 1 . For example, we could have a three-dimensional vector space with one dimension $|b l u e\rangle$ corresponding to blue sky, one dimension $\mid$ cloudy $\rangle$ corresponding to cloudy sky, and one dimension $\mid$ night $\rangle$ corresponding to night time, and suppose we define an observable $D$ to ask the question 'is it daytime?' $D$ has eigenvectors $|b l u e\rangle$ and $|c l o u d y\rangle$ with eigenvalue 1 (corresponding to yes) and eigenvector $\mid$ night $\rangle$ with eigenvalue 0 (corresponding to no). Then,

$$
D=1 \cdot \mid \text { blue }\rangle\langle\text { blue }|+1 \cdot| \text { cloudy }\rangle\langle\text { cloudy }|+0 \cdot| \text { night }\rangle\langle\text { night }|=| \text { blue }\rangle\langle\text { blue }|+| \text { cloudy }\rangle\langle\text { cloudy }|
$$

The probability of an answer 'yes' to the question 'is it daytime' is again the square of the probability amplitude, which is the magnitude of the projection onto the 'daytime' subspace spanned by the eigenvectors 


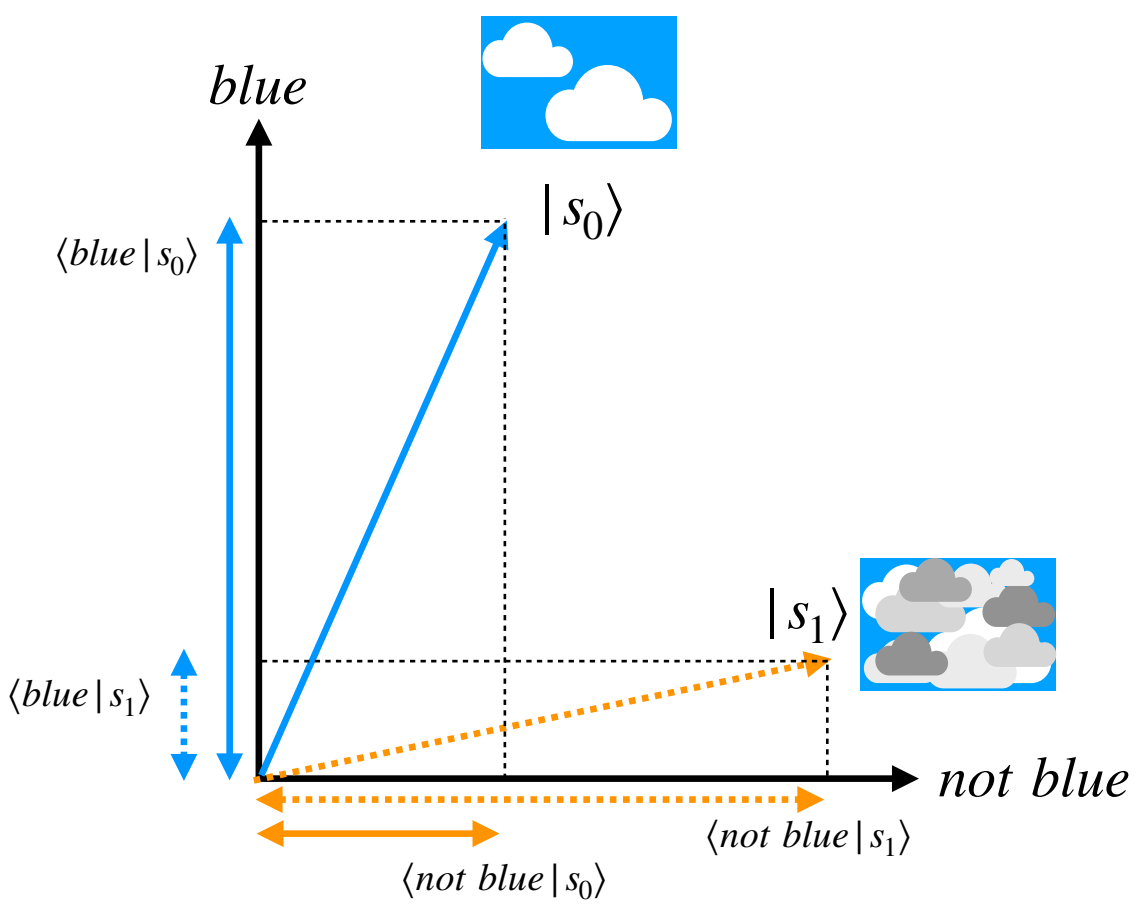

Figure 4: A 2-dimensional space with orthonormal basis $|b l u e\rangle, \mid$ not blue $\rangle$. The state $\left|s_{0}\right\rangle$ represents belief in a mostly blue sky with a couple of clouds, and the state $\left|s_{1}\right\rangle$ represents belief in a mostly grey sky. The projection onto the blue axis is high for $\left|s_{0}\right\rangle$ and low for $\left|s_{1}\right\rangle$, whereas the projection onto the not blue axis is low for $\left|s_{0}\right\rangle$ and higher for $\left|s_{1}.\right\rangle$ 
$|b l u e\rangle$ and $|c l o u d y\rangle$. If the current state of affairs is given by $|s\rangle$, then this is calculated by

$$
P\left(\text { yes }_{\text {day }}\right)=\langle s|D| s\rangle
$$

illustrated in figure 5

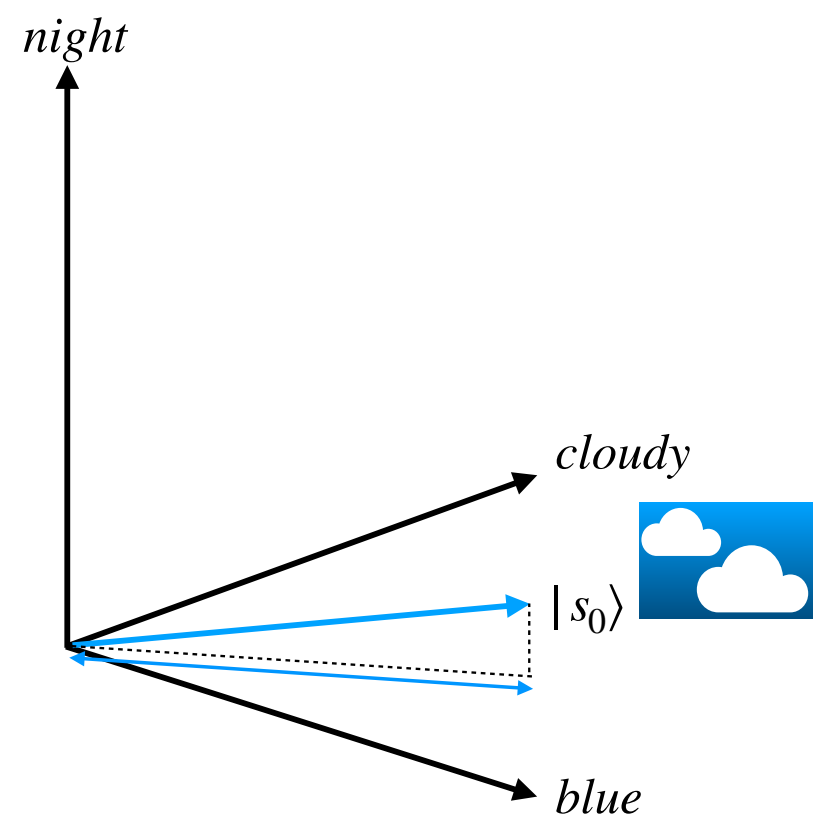

Figure 5: This figure shows a 3-dimensional space with orthonormal basis $\mid$ blue $\rangle, \mid$ cloudy $\rangle, \mid$ night $\rangle$. The state $\left|s_{0}\right\rangle$ represents belief that the sky is slightly cloudy and just starting to get dark. The probability amplitude of the answer 'yes!' to the question 'is it daytime' is given by the projection of $\left|s_{0}\right\rangle$ onto the plane spanned by $|b l u e\rangle,|c l o u d y\rangle$

More generally again, the current state of affairs may be uncertain. Quantum theory models this as a mixture of states

$$
\rho=\sum_{i} s_{i}\left|s_{i}\right\rangle\left\langle s_{i}\right|
$$

where $\sum_{i} s_{i}=1$.

Then the probability of the answer 'yes!' to the question 'is it daytime?' is given by

$$
\operatorname{Tr}(D \rho)
$$

where $\operatorname{Tr}$ stands for matrix trace. If we are not at all uncertain about the state, then $\rho=|s\rangle\langle s|$ and this is equivalent to the formula (12).

A key difference between classical probability theory and quantum probability theory is the treatment of conjunctions of events. In classical probability theory, the probability $P(A B)$ conjunction of two events $A$ and $B$ is equal to the probability $P(B A)$ of the two events in the opposite order. In quantum probability this does not hold, due to the the fact that in general the matrices corresponding to the observables $\hat{A}$ and $\hat{B}$ will not commute.

This means that conjunction in quantum probability needs to be defined in sequential terms. Specifically, suppose we want to calculate the probability an agent in state $|s\rangle$ judging that $A$ and then $B$. We have:

$$
P(A \text { and then } B)=P(A) \cdot P(B \mid A)
$$


The first term $P(A)$ is expressed as

$$
P(A)=\| A|s\rangle \|^{2}
$$

and the second term $P(B \mid A)$ is given by

$$
P(B \mid A)=\| B\left|s_{A}\right\rangle \|^{2}
$$

where $\left|s_{A}\right\rangle$ is the state of knowledge on the agent after judging that $A$ holds, i.e.

$$
\left|s_{A}\right\rangle=\frac{A|s\rangle}{\| A|s\rangle \|}
$$

Putting this all together we see that

$$
\begin{aligned}
P(A \text { and then } B) & =\| A|s\rangle\left\|^{2}\right\| B\left|s_{A}\right\rangle \|^{2} \\
& =\| A|s\rangle\left\|^{2}\left(\frac{B A|s\rangle}{\| A|s\rangle \|}\right)^{2}=\right\| B A|s\rangle \|^{2}
\end{aligned}
$$

i.e., the agent projects first to subspace $A$, and then to subspace $B$. In general, this is not equal to $\| A B|s\rangle \|^{2}$, as it would be in classical probability.

\subsection{Similarity judgements}

In Pothos et al. (2013), the model described above is used to account for the asymmetry of similarity judgements. Recall that Tversky (1977) showed that when asked to consider the similarity of one entity, such as a country, to another, the judgements of similarity are higher if the entity that is more salient ifs the one being compared to. So for example more participants agreed with the statement "Korea is similar to China" than "China is similar to Korea" (the participants were all Israeli college students).

Pothos et al. (2013) explicate this as follows. The entities being judged, for example China and Korea are modelled as subspaces of a given vector space. Each of these subspaces has an associated projector $P_{\text {China }}, P_{\text {Korea }}$. The state of belief of the agent is modelled as a quantum state $|\psi\rangle$. The probability that the agent's belief state is consistent with the concept China or the concept Korea is given by the square of the projection onto the relevant subspace: $\| P_{\text {China }} \mid$ psi $\rangle \|^{2}=\left\langle\psi \mid P_{\text {China }} \psi\right\rangle$. The initial belief state $\mid$ psi $\rangle$ is set up so that $\left\langle\psi \mid P_{\text {China }} \psi\right\rangle=\left\langle\psi \mid P_{\text {Korea }} \psi\right\rangle$.

Then, the similarity of Korea to China is calculated but first projecting $|p s i\rangle$ onto the Korea subspace, and then projecting the resulting vector onto the China subspace:

$$
\operatorname{Sim}(\text { Korea }, \text { China })=\| P_{\text {China }} P_{\text {Korea }}|\psi\rangle \|^{2}
$$

This quantity calculates the probability that the original state $|\psi\rangle$ is consistent with Korea, multiplied by the probability that this new state is consistent with China. The agent projects their belief state onto Korea, after which their new belief state is normalized, so that $\left|\psi_{\text {Korea }}\right\rangle=\frac{P_{\text {Korea }}|\psi\rangle}{\| P_{\text {Korea }}|\psi\rangle \|}$. This new state is projected onto China, and is consistent with the concept China with probability $\| P_{\text {China }}\left|\psi_{\text {Korea }}\right\rangle$. So we have that

$$
\| P_{\text {China }}\left|\psi_{\text {Korea }}\right\rangle\left\|^{2} \cdot\right\| P_{\text {Korea }}|\psi\rangle\left\|^{2}\right\| P_{\text {China }} P_{\text {Korea }}|\psi\rangle \|^{2}
$$

Now, as long as the projectors $P_{\text {China }}$ and $P_{\text {Korea }}$ do not commute, we have that $\operatorname{Sim}($ Korea, China $) \neq$ Sim (China, Korea. Moreover, Pothos et al. (2013) show that the similarity of Korea to China can be made to be higher than the similarity of China to Korea by modelling the more salient entity, in this case China, as a larger (higher dimensionality) subspace. 


\subsection{Diagnosticity}

Pothos et al. (2013) also show that the phenomenon of diagnosticity (section 2.2) can be modelled. In this model, each country is modelled as a single-dimensional subspace. The diagnosticity effect is produced by the order in which the projectors for the countries are applied to the agent's state vector $|\psi\rangle$.

Recall from section 2.2 that the diagnosticity effect says that similarity judgements can be affected but the context they are made in. An example from Tversky (1977) is the following. Take the sets of countries \{Austria, Sweden, Poland, Hungary\} and \{Austria, Sweden, Norway, Hungary\}. Participants see only one quadruple and are asked to judge which country Austria is most similar to. In the quadruple \{Austria, Sweden, Poland, Hungary\}, Austria is judged most similar to Sweden by a majority of participants. However, in $\{$ Austria, Sweden, Norway, Hungary\}, Austria is judged most similar to Hungary. Given that both are available in each set of countries, why does the similarity change?

Pothos et al. (2013) set each country to be represented as a ray. The similarity of Austria to any of the other countries in the set is modelled by saying that the agent's belief state $|\psi\rangle$ first projects onto the subspaces corresponding to the other countries. There are two orders in which this can happen, since there are two other countries. Therefore, these orders are averaged. Then, depending on the placing of the rays in the Hilbert space, the required effect can be produced. Pothos et al. (2013) show that with some reasonable assumptions about the similarities between the pairs of countries (i.e., we assume that Sweden is similar to Norway, and that Hungary is similar to Poland), the effect can be reliably reproduced over a number of simulations.

\subsection{Conjunction fallacy and over/under extension}

Franco (2009) provide an account of the conjunction fallacy using the notion of interference effect. The model works as follows. Recall that in the conjunction fallacy (section 2.3) the problem is that the conjunction of two events (that Linda is a bank teller and a feminist) is judged more probable than the single event that Linda is a feminist. According to classical probability this cannot hold.

Franco (2009); Busemeyer et al. (2011) model this using the quantum formalism described in section 3.1. They consider the two questions $A$ : 'is Linda a feminist?' and $B$ :'is Linda a bank teller?'. The question $A$ is represented by an observable on a 2-dimensional Hilbert space with eigenvectors $\left|a_{0}\right\rangle$ and $\left|a_{1}\right\rangle$ which have the eigenvalues $a_{0}=0$ (Linda is not a feminist) and $a_{1}=1$ (Linda is a feminist) respectively. Question $B$ is similarly represented. Furthermore, Franco (2009) represent the probability $P\left(b_{j} \mid a_{i}\right)$ as the probability that the answer to question $B$ is $b_{j}$, given that the answer to $A$ has already been determined to be $a_{i}$. If this latter condition is the case, then within this two-dimensional setting this means that the current state we are in is $\left|a_{i}\right\rangle$ itself, and therefore $P\left(b_{j} \mid a_{i}\right)=\left|\left\langle b_{j} \mid a_{i}\right\rangle\right|^{2}$, following equation (10). Notice that this means that $P\left(b_{j} \mid a_{i}\right)=\left|\left\langle b_{j} \mid a_{i}\right\rangle\right|^{2}=\left|\left\langle a_{i} \mid b_{j}\right\rangle\right|^{2}=P\left(a_{i} \mid b_{j}\right)$. Now as in equation (20), the probability of the answer that Linda is a feminist and a bank teller, $P\left(a_{1}-a n d-b_{1}\right)$ is modelled as $P\left(a_{1}\right) P\left(b_{1} \mid a_{1}\right)$.

Franco (2009) then lays out a specific relation between the eigenvectors of $\hat{A}$ and the eigenvectors of $\hat{B}$, as follows.

The two observables $\hat{A}$ and $\hat{B}$ both operate on the same two-dimensional Hilbert space, and the eigenvectors of $\hat{A}$ can be transformed into the eigenvectors of $\hat{B}$ via a unitary transformation

$$
U=\left(\begin{array}{cc}
e^{-i \xi \sqrt{P\left(b_{1} \mid a_{1}\right)}} & e^{-i \phi \sqrt{1-P\left(b_{1} \mid a_{1}\right)}} \\
-e^{i \phi \sqrt{1-P\left(b_{1} \mid a_{1}\right)}} & e^{i \xi \sqrt{P\left(b_{1} \mid a_{1}\right)}}
\end{array}\right)
$$

Visually, we have a pair of orthonormal bases as given in figure 6, where $\left|b_{i}\right\rangle=U\left|a_{i}\right\rangle$

Now, given this representation, the state of knowledge of the agent is represented as a vector:

$$
|s\rangle=\sqrt{P\left(a_{0}\right)}\left|a_{0}\right\rangle+e^{i \phi_{a}} \sqrt{P\left(a_{1}\right)}\left|a_{1}\right\rangle
$$

which gives the correct probabilities for the agent to judge whether or not Linda is a feminist.

Expressing this vector in the basis of $\hat{B}$, we obtain 


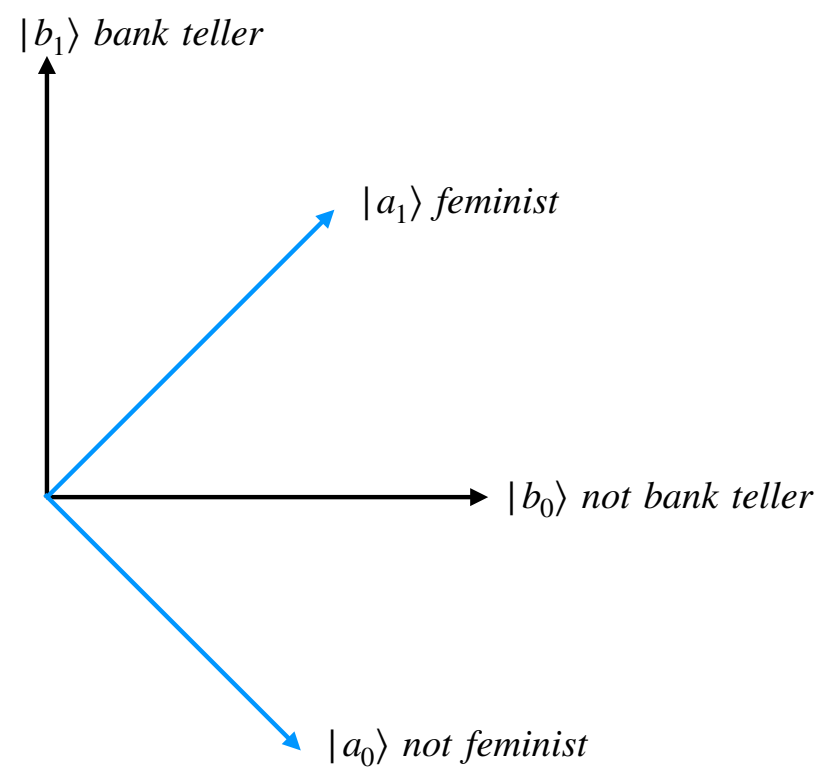

Figure 6: Orthonormal bases in a 2 dimensional space representing the questions 'Is Linda a bank teller?' and 'Is Linda a feminist?'

$$
\begin{aligned}
|s\rangle & =\sqrt{P\left(a_{0}\right)} U^{T}\left|b_{0}\right\rangle+\sqrt{P\left(a_{1}\right)} U^{T}\left|b_{1}\right\rangle \\
& =\left(\sqrt{P\left(a_{0}\right)} \sqrt{P\left(b_{1} \mid a_{1}\right)} e^{i \xi}-\sqrt{P\left(a_{1}\right)} \sqrt{P\left(b_{1} \mid a_{0}\right)} e^{i \phi_{a}-\phi}\right)\left|b_{0}\right\rangle \\
& +\left(\sqrt{P\left(a_{0}\right)} \sqrt{P\left(b_{1} \mid a_{0}\right)} e^{i \phi}+\sqrt{P\left(a_{1}\right)} \sqrt{P\left(b_{1} \mid a_{1}\right)} e^{i \phi_{a}-\xi}\right)\left|b_{1}\right\rangle
\end{aligned}
$$

Now, calculating $P\left(b_{1}\right)$, i.e. the probability that the agent judges that Linda is a bank teller, we get

$$
\begin{aligned}
P\left(b_{1}\right) & =\left|\sqrt{P\left(a_{0}\right)} \sqrt{P\left(b_{1} \mid a_{0}\right)} e^{i \phi}+\sqrt{P\left(a_{1}\right)} \sqrt{P\left(b_{1} \mid a_{1}\right)} e^{i \phi_{a}-\xi}\right|^{2} \\
& =P\left(a_{0}\right) P\left(b_{1} \mid a_{0}\right)+P\left(a_{1}\right) P\left(b_{1} \mid a_{1}\right)+2 \sqrt{P\left(a_{0}\right) P\left(a_{1}\right) P\left(b_{1} \mid a_{0}\right) P\left(b_{1} \mid a_{1}\right)} \cos \left(\phi+\xi+\phi_{a}\right)
\end{aligned}
$$

The last term in this sum is called the interference term $I(s, A)$. If $P\left(a_{0}\right) P\left(b_{1} \mid a_{0}\right)+I(s, A)$ is negative, then we obtain that $P\left(b_{1}\right)<P\left(a_{1}\right.$ and then $\left.b_{1}\right)$, i.e. that the probability that Linda is a bank teller is judged less likely than the probability that Linda is a feminist and a bank teller. This can be understood visually in figure 7 .

This calculation is expressed succinctly in Busemeyer et al. (2011) as follows. Consider that the event of answering 'yes' to 'is Linda a feminist' is represented by the projector $P_{F}$, and that the event of answering 'yes' to 'is Linda a bank teller' is given by $P_{B}$. The projectors corresponding to the answer 'no' to each question are given by $P_{\neg F}=1-P_{F}$ and $P_{\neg B}=1-P_{B}$ respectively.

Now, as in equation (20), given a state $|s\rangle$ we have

$$
P(F \text { and then } B)=\| P_{B} P_{F}|s\rangle \|^{2}
$$

We can compare this value with the value $P(B)=\| P_{B}|s\rangle \|^{2}$ as follows:

$$
\begin{aligned}
\| P_{B}|s\rangle \|^{2} & =\| P_{B} \cdot I|s\rangle\left\|^{2}=\right\| P_{B}\left(P_{F}+P_{\neg F}|s\rangle \|^{2}\right. \\
& =\| P_{B} P_{F}|s\rangle+P_{B} P_{\neg F}|s\rangle \|^{2} \\
& =\| P_{B} P_{F}|s\rangle\left\|^{2}+\right\| P_{B} P_{\neg F}|s\rangle \|^{2}+\left\langle s_{B \neg F} \mid s_{B F}\right\rangle+\left\langle s_{B F} \mid s_{B \neg F}\right\rangle
\end{aligned}
$$




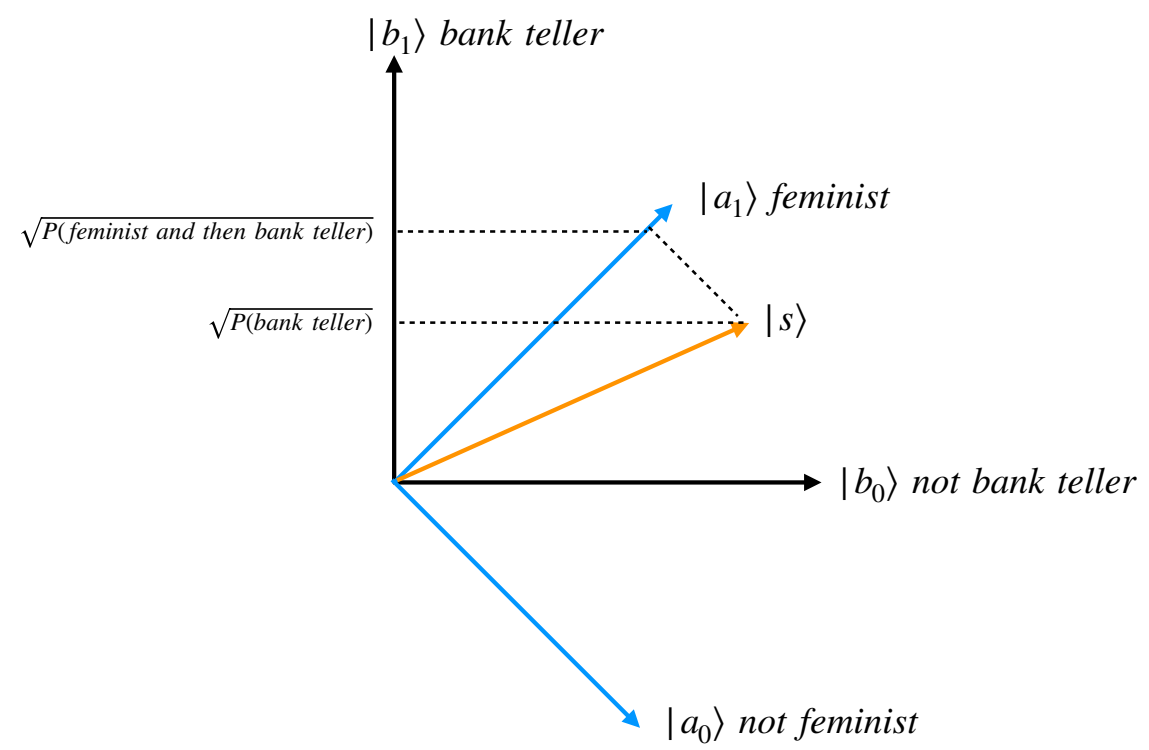

Figure 7: Starting in the state $|s\rangle$, the probability that Linda is judged to be a bank teller is less that the probability that Linda is judged first to be a feminist and then to be a bank teller

where $\left|s_{B F}\right\rangle=P_{B} P_{F}|s\rangle$ and $\left|s_{B \neg F}\right\rangle=P_{B} P_{\neg F}|s\rangle$.

This term $\left\langle s_{B \neg F} \mid s_{B F}\right\rangle+\left\langle s_{B F} \mid s_{B \neg F}\right\rangle$ is the interference term that we called $I(s, A)$ above and we can construct this term to be negative. Again, if $\left\langle s_{B \neg F} \mid s_{B F}\right\rangle+\left\langle s_{B F} \mid s_{B \neg F}\right\rangle+\| P_{B} P_{\neg F}|s\rangle \|^{2}$ is negative overall, then we have

$$
\| P_{B}|s\rangle\left\|^{2}<\right\| P_{B} P_{F}|s\rangle \|^{2}
$$

and the conjunction fallacy is fulfilled.

A related phenomenon to the conjunction fallacy is that a similar phenomenon is observed when considering disjunctions. Morier and Bordiga (1984) show that in a setup where participants are asked to judge the probability that Linda is a) a feminist, b) a bank teller, c) a feminist and a bank teller and d) a feminist or a bank teller, the order from least probable to most probable is given by

$$
P(\text { bank teller })<P(\text { feminist and bank teller })<P(\text { feminist or bank teller })<P(\text { feminist })
$$

Busemeyer et al. (2011) show that their probability model can also account for this phenomenon. They consider the probability of answering 'no' to the question 'is Linda neither a bank teller nor a feminist?'. This is modelled as the probability $1-\left\|P_{\neg F} P_{\neg B}\right\|^{2}$. Notice that here, the order of the projectors is such that the state is first projected onto $P_{\neg B}$ - this is because this subspace is more probable. Consider

$$
\| P_{\neg F}|s\rangle\left\|^{2}=\right\| P_{\neg F} P_{\neg B}\left\|^{2}+\right\| P_{\neg F} P_{B} \|^{2}+\left\langle s_{\neg F B} \mid s_{\neg F \neg B}\right\rangle+\left\langle s_{\neg F \neg B} \mid s_{\neg F B}\right\rangle
$$

Again, if the interference term $\left\langle s_{\neg F B} \mid s_{\neg F \neg B}\right\rangle+\left\langle s_{\neg F \neg B} \mid s_{\neg F B}\right\rangle$ is sufficiently negative, we have $\left\|P_{\neg F} P_{B}\right\|^{2}+$ $\left\langle s_{\neg F B} \mid s_{\neg F \neg B}\right\rangle+\left\langle s_{\neg F \neg B} \mid s_{\neg F B}\right\rangle<0$ and then $\| P_{\neg F}|s\rangle\left\|^{2}<\right\| P_{\neg F} P_{\neg B} \|^{2}$, as needed for the disjunction fallacy.

Double overextension Whilst this model covers a number of kinds of probability judgement error, one phenomenon that is not covered is the phenomenon of double overextension. This occurs when the probability of an item belonging to a conjunction of concepts is judged to be higher than the probability of the item belonging to either of the constituent concepts. In the Linda story, this would mean that the probability of Linda being judged to be a feminist and a bank teller would not only be judged higher than the probability of her being a bank teller, but also higher than her being judged to be a feminist. 
A quick look at the formalism shows that this cannot hold, at least as long as we assume the the order of conjunction is fixed.

$$
\begin{aligned}
P(F \text { and then } B) & =P(F) \cdot P(B \mid F)=\| P_{B} P_{F}|s\rangle \|^{2} \\
& =\| P_{B}\left|s_{F}\right\rangle\left\|^{2} \cdot\right\| P_{F}|s\rangle\left\|^{2}<\right\| P_{F}|s\rangle \|^{2}=P(F)
\end{aligned}
$$

As long as we hold the order fixed, we cannot have that both $P(F)$ and $P(B)$ are lower than $P(F$ and then $B)$

However, this kind of double overextension has been empirically observed, as described in section 2.6. In section 3.8 we will describe an approach in which the problem of double overextension can be modelled.

\subsection{Violation of the 'sure thing' principle}

The 'sure thing' principle states that if an agent would take a specific course of action $C$ in all possible states of the world, then they should take action $C$ when they do not know what the state of the world is. However, as described in 2.5, humans usually do not behave like this. This is seen in a prisoner's dilemma situation, the situation where we are choosing whether to book a holiday, or a situation where we are choosing whether to make a bet, as well as many more. Pothos and Busemeyer (2009) show that this phenomenon can be modelled using the same kind of quantum similarity model described in sections 3.2. Other approaches include that in Khrennikov and Haven (2009).

The model is applied to a prisoner's dilemma situation. The situation can be modelled as the tensor product of one space modelling our opponents actions, and one space modelling our actions. Each space is two-dimensional, with one dimension corresponding to the 'cooperate' $(C)$ action, and the other corresponding to the 'defect' $(D)$ action. The agent's state at the start of the game is therefore represented as the state

$$
\left|\psi_{0}\right\rangle=\frac{1}{2}|D D\rangle+|D C\rangle+|C D\rangle+|C C\rangle
$$

If the opponent is known to defect, then the state changes to

$$
\left|\psi_{D}\right\rangle=\alpha_{D D}|D D\rangle+\alpha_{D C}|D C\rangle
$$

where $\alpha_{D D}^{2}+\alpha_{D C}^{2}=1$. On the other hand, if the opponent is know to cooperate, the state changes to

$$
\left|\psi_{C}\right\rangle=\alpha_{C D}|C D\rangle+\alpha_{C C}|C C\rangle
$$

where $\alpha_{C D}^{2}+\alpha_{C C}^{2}=1$. Our decision whether or not to defect is modelled as a projection $M$ onto our defect axis, i.e.

$$
M=|D D\rangle\langle D D|+| C D\rangle\langle C D|
$$

Now, if we know that our opponent defected, then our probability of defection is:

$$
\begin{aligned}
\left\langle\psi_{D}|M| \psi_{D}\right\rangle & =\left(\alpha _ { D D } ^ { * } \left\langleD D\left|+\alpha_{D C}^{*}\langle D C|\right)(|D D\rangle\langle D D|+| C D\rangle\langle C D|)\left(\alpha_{D D}|D D\rangle+\alpha_{D C}|D C\rangle\right)\right.\right. \\
& =\left|\alpha_{D D}\right|^{2}
\end{aligned}
$$

where $|\alpha|$ indicates the absolute value of $\alpha$ However, if we know that our opponent cooperated, then our probability of defection is:

$$
\begin{aligned}
\left\langle\psi_{C}|M| \psi_{C}\right\rangle & =\left(\alpha _ { C D } ^ { * } \left\langleC D\left|+\alpha_{C C}^{*}\langle C C|\right)(|D D\rangle\langle D D|+| C D\rangle\langle C D|)\left(\alpha_{C D}|C D\rangle+\alpha_{C C}|C C\rangle\right)\right.\right. \\
& =\left|\alpha_{C D}\right|^{2}
\end{aligned}
$$

We model not knowing whether the opponent has defected or not as the superposition $|\psi\rangle=\frac{1}{\sqrt{2}}\left(\left|\psi_{D}\right\rangle+\left|\psi_{C}\right\rangle\right)$ When we are in this state of ignorance, we have:

$$
\begin{aligned}
\langle\psi|M| \psi\rangle= & \frac{1}{2}\left(\alpha_{D D}^{*}\langle D D|+\alpha_{D C}^{*}\langle D C|+\alpha_{C D}^{*}\langle C D|+\alpha_{C C}^{*}\langle C C|\right)(|D D\rangle\langle D D|+| C D\rangle\langle C D|) \\
& \left(\alpha_{D D}|D D\rangle+\alpha_{D C}|D C\rangle+\alpha_{C D}|C D\rangle+\alpha_{C C}|C C\rangle\right) \\
= & \frac{1}{2}\left(\alpha_{D D}^{2}+\alpha_{D C}^{2}\right)+\operatorname{Re}\left(\left\langle\alpha_{D D} D D \mid \alpha_{D C} D C\right\rangle\right)
\end{aligned}
$$


Again, this last term is an interference term, and may be negative. This means that we can obtain the situation that the probability of defecting is smaller in the state of ignorance than it is in the case that we know what the action of our opponent is.

\subsection{Question order models and contextuality}

The quantum probability theory of judgement proposed in Busemeyer et al. (2011) and described in section 3.1 is applied to question order effects in Wang et al. (2013). The question order effects are those taken from Moore (2002), outlined in section 2.4. Consider the consistency effect exemplified by the questions 'Do you think Clinton is trustworthy?' and 'Do you think Gore is trustworthy?'. The consistency effect means that when the question is asked second (the comparative context), people change their responses to make them more similar to the response to the first question, in comparison to when the question is asked first. Wang et al. (2013) model this as follows.

A person's belief is represented by a state vector $|s\rangle$ in an $n$-dimensional Hilbert space. Each potential response $x$ to a question $Q$ corresponds to a subspace $Q x$ that has a unique orthogonal projector $P_{Q x}$. The projectors to all responses to question $Q$ sum to the identity: $\sum_{x} P_{Q x}=I$. The probability of responding $x$ to the question $Q$ is given by $\left\|P_{Q x}\right\|^{2}$, and the updated belief state after deciding on an answer to the question is $\left|s_{Q x}\right\rangle=\frac{P_{Q x}|s\rangle}{\| P_{Q x}|s\rangle \mid}$. As in 3.1 , the probability of responding $x$ to question $A$ and then $y$ to question $B$ is:

$$
p(A x \text { and then } B y)=\| P_{A x}|s\rangle\left\|^{2}\right\| P_{B y}\left|s_{A x}\right\rangle\left\|^{2}=\right\| P_{B y} P_{A x}|s\rangle \|^{2}
$$

An order effect for a given question occurs when the probability of giving an answer to a question in the comparative context differs from the probability of giving that answer in the non-comparative context. In the Clinton/Gore setting, we have that the total probability $T P_{C y}$ of answering 'yes' to the question 'is Clinton trustworthy' in the comparative context is given by:

$$
T P_{C y}=p(G y \text { and then } C y)+p(G n \text { and then } C y)=\| P_{C} P_{G}|s\rangle\left\|^{2}+\right\| P_{C} P_{\neg G}|s\rangle \|^{2}
$$

However, as we saw in 3.4, the probability of answering 'yes' in th non-comparative context is given by:

$$
\begin{aligned}
p(C y) & \left.=\| P_{C}|s\rangle\left\|^{2}=\right\| P_{C} P_{G}|s\rangle+P_{C} P_{\neg G}\right)|s\rangle \|^{2} \\
& \left.=\| P_{C} P_{G}|s\rangle\left\|^{2}+\right\| P_{\neg G}\right)|s\rangle \|^{2}+2 \operatorname{Re}\left\langle s\left|P_{G} P_{c}\left(1-P_{G}\right)\right| s\right\rangle \quad=T P_{C} y+2 \operatorname{Re}\left\langle s\left|P_{G} P_{c}\left(1-P_{G}\right)\right| s\right\rangle
\end{aligned}
$$

The order effect $C_{C}$ is therefore given by

$$
\begin{aligned}
T P_{C y}-p(C y) & =-2 \operatorname{Re}\left\langle s\left|P_{G} P_{C}\left(1-P_{G}\right)\right| s\right\rangle=2 \| P_{C} P_{G}|s\rangle \|^{2}-2 \operatorname{Re}\left\langle s\left|P_{G} P_{C}\right| s\right\rangle \\
& =2 \| P_{C} P_{G}|s\rangle \|^{2}-2\left|\left\langle s\left|P_{G} P_{C}\right| s\right\rangle\right| \cos \phi \\
& =2 \| P_{C} P_{G}|s\rangle\left\|^{2}-2 \theta\right\| P_{C}|s\rangle\|\cdot\| P_{G}|s\rangle \|
\end{aligned}
$$

where $\theta=\cos \phi\left|\left\langle s\left|P_{G} P_{C}\right| s\right\rangle\right| /\left(|| P_{C}|s\rangle\|\cdot\| P_{G}|s\rangle||\right)$.

A similar calculation gives the order effect for Gore, $C_{G}$ to be:

$$
C_{G}=2 \| P_{G} P_{C}|s\rangle\left\|^{2}-2 \theta\right\| P_{C}|s\rangle\|\cdot\| P_{G}|s\rangle \|
$$

Now, since $\theta$ is the same in both cases, we have:

$$
\begin{aligned}
0 & =2 p(G y \text { and then } C y)-C_{C}-\left(2 p(C y \text { and then } G y)-C_{G}\right) \\
& =2 p(G y \text { and then } C y)-p(G y \text { and then } C y)-p(G n \text { and then } C y)+p(C y) \\
& -(2 p(C y \text { and then } G y)-p(C y \text { and then } G y)-p(C n \text { and then } G y)+p(G y)) \\
& =p(G y \text { and then } C y)-p(G n \text { and then } C y)+p(C y \text { and then } G y)+p(C y \text { and then } G n) \\
& -p(C y \text { and then } G y)+p(C n \text { and then } G y)-p(G y \text { and then } C y)-p(G y \text { and then } C n) \\
& =p(C y \text { and then } G n)+p(C n \text { and then } G y)-p(G y \text { and then } C n)-p(G n \text { and then } C y)
\end{aligned}
$$


This last quantity $p(C y$ and then $G n)+p(C n$ and then $G y)-p(G y$ and then $C n)-p(G n$ and then $C y)$ is termed $q$.

More generally, take the quantity $p_{A B}=p(A y$ and then $B n)+p(A n$ and then $B y)$ to be the probability that the answers to the two questions are different in the $A B$ order, and take $p_{B A}=p(B y$ and then $A n)+$ $p(B n a n d$ then $A y)$ in the $B A$ order. Then the quantum model predicts that

$$
q=p_{A B}-p_{B A}=0
$$

Wang et al. (2013) test this theory on the data from Moore (2002) (described in section 2.4) using the sample frequencies to compute the values $p_{A B}$ and $p_{B A}$, and then using a $z$-test to compute whether $q$ is significantly different from 0 .

The conclusions are that the quantum question-order model fits the data extremely well. The three datasets exemplifying the consistency, contrast, and additive effects have $q$-values that are not at all significantly different from 0 . The fourth dataset (Rose-Jackson) was not well modelled by the QQ model. However, the authors argue that there is a violation of the assumptions in this model. Specifically, in this case, additional information is supplied about each of the characters, and this entails that the value $\theta$ is not the same in both cases. Hence, the cancellations worked out in equation (56) do not hold in this case.

A crucial part of the argument in this paper is also that other probabilistic models cannot account for both the question order effects seen and the QQ equality. In the case of a Bayesian probability model, the assumption is that the event $A y$ is a set contained in a sample space, and that $B y$ is another set contained in the same sample space. The probability of the conjunction is then defined by the intersection of the two events, which is commutative. In order to introduce order effects, two new events must be introduced: an event $O 1$ : 'A was asked before $\mathrm{B}$ ', and an event $O 2$ : 'B was asked before $\mathrm{A}$ '. However, in general, introducing these two events does not predict the QQ equality to obtain.

Markov models can incorporate question order effects, since they can encode the memory of previous events into the state representation. However, the authors argues that in general, the QQ equality is not upheld.

\subsection{Contextuality}

A fundamental aspect of quantum mechanics is contextuality. The phenomenon of contextuality is as follows. Suppose we have a system with a number of agents who can perform measurements on the system, and suppose that we have determined joint distributions on the outcomes of pairs of measurements, by means of redoing the experiment a number of times. Contextuality occurs when it is not possible to form a joint distribution across all measurement outcomes such that this joint distribution agrees with the pairwise distributions. This means that before the measurement happens, the outcome of the measurement is undetermined. Contrast this with the outcomes of measurements in normal life. Suppose you wake up, and you wonder about the weather outside. You can take a measurement (open your curtains) and see that it is sunny, or cloudy. However, whether or not it was sunny or cloudy was determined before you opened the window.

We explain this below following the explanation in Nielsen and Chuang (2010). To show that the joint distribution does not exist, an analysis of the situation outside of quantum theory is made, and a Bell inequality is formed. Suppose that we have a system and two observers Alice and Bob. Alice can make two measurements, and each has the possibility of returning 1 or -1 . Bob can also make two measurements, and each of these has the possibility of returning 1 or -1 . The measurements are each modelled as random variables $A, A^{\prime}, B, B^{\prime}$. We now consider the quantity

$$
A B+A^{\prime} B+A^{\prime} B^{\prime}-A B^{\prime}=\left(A+A^{\prime}\right) B+\left(A^{\prime}-A\right) B^{\prime}
$$

Since $A, A^{\prime}= \pm 1$, either $\left(A+A^{\prime}\right) B=0$ or $\left(A^{\prime}-A\right) B^{\prime}=0$. And then we have that $A B+A^{\prime} B+A^{\prime} B^{\prime}-A B^{\prime}=$ \pm 2 . We now consider what is termed a hidden variable. We suppose that it is possible to determine the outcome of each of the random variables individually so that $A=a, A^{\prime}=a^{\prime}, B=b$, and $B^{\prime}=b^{\prime}$, giving us 
a joint probability $p\left(a, a^{\prime}, b, b^{\prime}\right)$. Now, we can calculate the expected value of $A B+A^{\prime} B+A^{\prime} B^{\prime}-A B^{\prime}$ as

$$
\begin{aligned}
\mathbb{E}\left(A B+A^{\prime} B+A^{\prime} B^{\prime}-A B^{\prime}\right) & =\sum_{a, a^{\prime}, b, b^{\prime}} p\left(a, a^{\prime}, b, b^{\prime}\right)\left(a b+a^{\prime} b+a^{\prime} b^{\prime}-a b^{\prime}\right) \\
& \leq 2 \sum_{a, a^{\prime}, b, b^{\prime}} p\left(a, a^{\prime}, b, b^{\prime}\right)=2
\end{aligned}
$$

However, within quantum theory this inequality is violated. Consider the quantum state $|p s i\rangle=\frac{|01\rangle+|10\rangle}{\sqrt{2}}$, and suppose Alice can perform measurements $A=Z_{1}$ or $A^{\prime}=X_{1}$ on the first qubit and Bob can perform measurements $B=\frac{-Z_{2}-X_{2}}{\sqrt{2}}$ or $B^{\prime}=\frac{Z_{2}-X_{2}}{\sqrt{2}}$ on the second qubit ${ }^{10}$. Calculating the average value $\langle M\rangle$ of an observable $M$ as $\langle\psi|M| \psi\rangle$, we find that:

$$
\langle A B\rangle=\frac{1}{\sqrt{2}}, \quad\left\langle A^{\prime} B\right\rangle=\frac{1}{\sqrt{2}}, \quad\left\langle A^{\prime} B^{\prime}\right\rangle=\frac{1}{\sqrt{2}}, \quad\left\langle A B^{\prime}\right\rangle=-\frac{1}{\sqrt{2}}
$$

meaning that $\mathbb{E}\left(A B+A^{\prime} B+A^{\prime} B^{\prime}-A B^{\prime}\right)=2 \sqrt{2}$, and violating the inequality in (64).

The violation of this inequality has been demonstrated experimentally, and shows that either there is no set of definite values of the random variables $A, A^{\prime}, B$, and $B^{\prime}$ that exist independent of our observation, or that the act of Alice's measurement affects the act of Bob's measurement.

The notion of contextuality also arises within cognitive science. Contextuality is crucially different from context-dependence. The phenomenon of context dependence arises when the answer to a question depends on the context that the question is asked in. This kind of context-dependence is called direct influences (Dzhafarov and Kujala, 2016). However, another kind of context-dependence that is analogous to quantum contextuality may be present. In Basieva et al. (2019) the following example is given. Suppose we have the three questions

1. Do you like chocolate?

2. Are you afraid of pain?

3. Do you see your dentist regularly?

Imagine that you are asked a pair of these questions. It may well be that the context in which you are asked a given question directly influences your answer of that question. So if you are asked 1) and 2) together, you may respond positively to 1), but if you are asked 1) and 3) together, the thought of going to the dentist may incline you to answer negatively to 1). This is the notion of direct influence. Basieva et al. (2019), following Dzhafarov and Kujala (2016) use the following notation: a response to question $q_{i}$ asked in context $c_{j}$ is a random variable denoted $R_{i}^{j}$, and described by:

$$
R_{i}^{j}: \begin{array}{cc|c}
\text { Yes } & \text { No } & \text { response } \\
\hline p_{i}^{j} & 1-p_{i}^{j} & \text { probability }
\end{array}
$$

These random variables can be laid out as follows (Dzhafarov and Kujala, 2016)

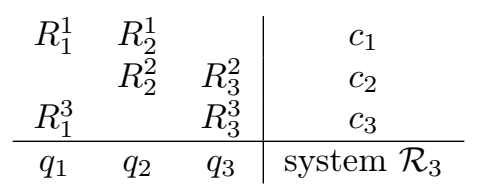

This says that in context $1\left(c_{1}\right)$, questions 1 and $2\left(q_{1}\right.$ and $\left.q_{2}\right)$ are asked, and so on.

\footnotetext{
${ }^{10} Z$ and $X$ are the Pauli matrices
} 
Now, we might assume that all direct influences have somehow been eliminated, so that you have the same probability of answering yes or no to a given question no matter what the context. We then have that

$$
\begin{aligned}
& R_{1}^{1}=R_{1}^{3} \\
& R_{2}^{1}=R_{2}^{2} \\
& R_{3}^{2}=R_{3}^{3}
\end{aligned}
$$

However, we can also imagine a setup in which, if you answer yes to one question in the context, you always answer no to the other question. Representing yes and no by 1 and -1, we then have:

$$
\begin{aligned}
& R_{1}^{1}=-R_{2}^{1} \\
& R_{2}^{2}=-R_{3}^{2} \\
& R_{1}^{3}=-R_{3}^{3}
\end{aligned}
$$

We can then easily derive a contradiction by following a chain of equalities:

$$
R_{1}^{1}=R_{1}^{3}=-R_{3}^{3}=-R_{3}^{2}=R_{2}^{2}=R_{2}^{1}=-R_{1}^{1}
$$

This is saying that the pairs of random variables $R_{1}^{1}$ and $R_{1}^{3}, R_{2}^{1}$ and $R_{2}^{2}, R_{3}^{2}$ and $R_{3}^{3}$ cannot be identical. This kind of context dependence is not reducible to direct influences.

Within quantum theory, contextuality applies only to systems in which there is no notion of direct influences. Measurements of a property in different conditions are described by the same random variable. The theory developed in Dzhafarov and Kujala (2016), called contextuality-by-default or CbD can also take account of the presence of direct influences, and is therefore an extension of the quantum-theoretic notion. $\mathrm{CbD}$ is able to quantify how much the distributions of responses to different questions differ in different contexts, and how much the identities of the random variables differ in different contexts. By comparing these two quantities, it is possible to show how much contextuality is present over and above the effect of direct influences.

In previous work examining the contextuality of human judgements, the impact of direct influences was not always considered (Khrennikov et al., 2014; Dzhafarov and Kujala, 2014; Aerts et al., 2013), and so it was not clear the extent to which true contextuality was actually present in human decision making. For example, in Bruza et al. (2012, 2015), whilst the effect of direct influences was taken into account, the presence of true contextuality was only minimally detected. However, in Basieva et al. (2019); Dzhafarov (2019); Cervantes and Dzhafarov (2019), experiments have been carried out to show that this phenomenon is indeed present in human judgement.

\subsection{Concept combination and overextension}

As described in 2.6, the phenomenon of overextension and underextension has been observed in experiments in Hampton (1987, 1988a,b); Smith and Osherson (1984).

In Aerts and Gabora (2005a,b) the authors set out a quantum model of concepts and their combination. The model of a concept is called the State-Context-Property or SCOP formalism. The idea behind this is that typicality ratings of exemplars in a concept can depend on the context in which they are considered. For example, in the context 'The pet is chewing a bone', a set of example might be ordered as: dog, cat, rabbit, hamster, guinea pig, mouse, hedgehog, bird, parrot, snake, canary, goldfish, spider, guppy, whereas under the context 'The pet is being taught', the same set of exemplars would be ordered dog, parrot, cat, bird, hamster, canary, guinea pig, rabbit, mouse, hedgehog, snake, goldfish, guppy, spider. The idea is that each context acts on the concept to bring it into another state.

A SCOP consists of a set $\Sigma$ of states $p_{i}$, a set $\mathcal{M}$ of contexts $e_{i}$, such as 'the pet is chewing a bone', and a set $\mathcal{L}$ of properties $a_{i}$, such as 'is feathered'. Together with these sets, there is a function

$$
\mu: \Sigma \times \mathcal{M} \times \Sigma \rightarrow[0,1]
$$


which is a probability function that describes how a state $p$ under the influence of context $e$ changes into state $q$.

$$
\mu(q, e, p)=\left\langle p\left|P_{e}\right| p\right\rangle
$$

There is also a function

$$
\nu: \Sigma \times \mathcal{L} \rightarrow[0,1]
$$

which gives the weight of a property $a$ for the concept in the state $p$. So, for example, after considering the concept 'pet' in the context 'the pet is chewing a bone', the weight of the property 'is furry' might increase.

$$
\nu(p, a)=\left\langle p\left|P_{a}\right| p\right\rangle
$$

In Aerts and Gabora (2005a), a Hilbert space representation of the theory is given. Concepts are represented in a Hilbert space in which the basis dimensions of the space represent basic states of the concept. The basic states of a concept are defined via basic contexts of a concept, which roughly means that the basic concepts contexts are the most specific. For example, we might have the contexts $a_{1}=$ 'the pet is chewing a bone', and $a_{2}=$ 'the pet is chewing a bone in the garden', and we would say that $a_{2}$ is more specific than $a_{1}$. The set of atomic contexts is denoted by $X$, and these are considered to be rank-1 projectors. The basic states are then the states corresponding to these rank-1 projectors. The ground state $\hat{p}$ of a concept is given by the following superposition of the basis states of the Hilbert space:

$$
|\hat{p}\rangle=\sum_{u \in X} \frac{1}{|X|}|u\rangle
$$

so that the ground state has an equal probability of changing to any basic state under one of the basic contexts.

General contexts are represented by projectors that are not merely of rank 1 . So suppose that we have 100 basic contexts, and 25 of these basic contexts are more specific than $e_{\text {bone }}=$ the pet is chewing a bone, i.e. they are more detailed contexts in which the pet is chewing a bone ${ }^{11}$. Then we would build a projector corresponding to the context $e_{\text {bone }}$ by summing together the projectors for the basic contexts in the set $E_{\text {bone }}=\left\{u \in X \mid u\right.$ is more specific than $\left.e_{\text {bone }}\right\}$ :

$$
P_{\text {bone }}=\sum_{u \in E_{\text {bone }}}|u\rangle\langle u|
$$

The new state under the influence of this context is calculated in the standard way, i.e. in the same way that the knowledge state of the agent is evolved in section 3.1, equation (18):

$$
\left|p_{\text {bone }}\right\rangle=\frac{P_{\text {bone }}|\hat{p}\rangle}{\| P_{\text {bone }}|\hat{p}\rangle \|}
$$

In our example with 100 basic contexts and 25 of these basic contexts are more specific than $e_{1}$, we have that

$$
\begin{aligned}
P_{\text {bone }}|\hat{p}\rangle & =\sum_{u \in E_{\text {bone }}}|u\rangle\langle u|| \hat{p}\rangle=\sum_{u \in E_{\text {bone }}} \frac{1}{\sqrt{100}}|u\rangle \\
\| P_{\text {bone }}|\hat{p}\rangle \|^{2} & =\left\langle\hat{p}\left|P_{\text {bone }}\right| \hat{p}\right\rangle=\sum_{u \in E_{\text {bone }}}|\langle\hat{p} \mid u\rangle|^{2}=\sum_{u \in E_{\text {bone }}} \frac{1}{100}=\frac{25}{100}
\end{aligned}
$$

so that

$$
\left|p_{\text {bone }}\right\rangle=\sum_{u \in E_{\text {bone }}} \frac{1}{\sqrt{25}}|u\rangle=\sum_{u \in E_{\text {bone }}} \frac{1}{5}|u\rangle
$$

\footnotetext{
${ }^{11}$ Note that there might be basic contexts that are unrelated to 'the pet is chewing a bone', for example 'the pet is chewing a cracker in a cage and scratching its wing' - although this might be a basic context, it is not related to the context 'the pet is chewing a bone'.
} 
Recall that in the pet fish problem the problem is that the exemplar 'goldfish' is viewed as more typical to the 'pet fish', conceived as the conjunction of two concepts 'pet' and 'fish' than it is to either of the constituent concepts. To describe the notion of the typicality of an exemplar to a concept, Aerts and Gabora (2005a) use the function $\mu$ described in equation (75). Aerts and Gabora (2005a) explicate the notion of typicality as follows. Each exemplar is assigned to a context, so if we want to consider the exemplar $p_{g f}=$ goldfish, we form the context $e_{g f}=$ the pet is a goldfish. Now, consider the concept pet in the context $e_{\text {bone }}=$ the pet is chewing a bone. As described by equation (18) and equation (84) in the example above, we apply the context to the state pet to obtain the state $p_{\text {bone }}$.

To calculate the weight of the exemplar goldfish in the context the pet is chewing a bone, we calculate the probability that $p_{\text {bone }}$ changes to $p_{g f}$ in the context $e_{g f}$, i.e. we calculate:

$$
\mu\left(p_{g f}, e_{g f}, p_{\text {bone }}\right)=\left\langle p_{\text {bone }}\left|P_{g f}\right| p_{\text {bone }}\right\rangle
$$

Earlier, we said that there are 25 out of 100 basic contexts in which the pet is chewing a bone. Suppose there are 10 contexts where the pet is a goldfish, but only 1 context where the pet is chewing a bone and is a goldfish. Then we will have

$$
\begin{aligned}
\mu\left(p_{g f}, e_{g f}, p_{\text {bone }}\right) & =\left\langle p_{\text {bone }}\left|P_{\text {gf }}\right| p_{\text {bone }}\right\rangle=\sum_{u \in E_{g f}} \sum_{v \in E_{\text {bone }}} \sum_{w \in E_{\text {bone }}} \frac{1}{25}\langle v \mid u\rangle\langle u \mid w\rangle \\
& =\sum_{u \in E_{\text {bone }} \cap E_{g f}} \frac{1}{25}=\frac{1}{25}
\end{aligned}
$$

So the weight of the exemplar goldfish in the context the pet is chewing a bone is $\frac{1}{25}$. This quantity is what Aerts et al. (2015) use to formalize the notion of typicality of an exemplar to a concept.

To examine the pet fish situation, we will first set up an example situation. In Aerts and Gabora (2005a), these figures were generated from a psychological experiment involving asking humans for the typicality figures. In this chapter, we simplify the figures for explanatory purposes.

Suppose that we have a pool of 100 basic states for pet and a pool of 50 basic states for fish. The basic states are distributed amongst the following exemplars as in table 6 below:

Table 6: Numbers of basic states assigned to each exemplar in the concepts pet and fish

\begin{tabular}{c|cc} 
& Pet & Fish \\
\hline dog & 50 & 0 \\
cat & 35 & 0 \\
goldfish & 10 & 10 \\
shark & 5 & 20 \\
tuna & 0 & 20
\end{tabular}

For each concept, we outline how many basic contexts are included in each of the contexts $e_{\text {pet }}^{x}=$ the $x$ is a pet, $e_{\text {fish }}^{x}=$ the $x$ is a fish, $e_{\text {furry }}^{x}=$ the $x$ is furry, $e_{\text {swim }}^{x}=$ the $x$ is swimming around in a glass tank in the house

Table 7: Numbers of basic contexts assigned to each context for each exemplar in the concept pet

\begin{tabular}{c|cccc} 
& $e_{\text {pet }}^{\text {pet }}$ & $e_{\text {fish }}^{\text {pet }}$ & $e_{\text {furry }}^{\text {pet }}$ & $e_{\text {swim }}^{\text {pet }}$ \\
\hline $\mathrm{dog}$ & 50 & 0 & 45 & 0 \\
cat & 35 & 0 & 30 & 0 \\
goldfish & 10 & 10 & 0 & 9 \\
shark & 5 & 5 & 0 & 5 \\
tuna & 0 & 0 & 0 & 0
\end{tabular}

The typicality of goldfish in the concept pet is then 
Table 8: Numbers of basic contexts assigned to each context for each exemplar in the concept fish

$$
\begin{array}{rl|cccc} 
& e_{\text {pet }}^{\text {fish }} & e_{\text {fish }}^{\text {fish }} & e_{\text {furry }}^{\text {fish }} & e_{\text {swim }}^{\text {fish }} \\
\hline \text { dog } & 0 & 0 & 0 & 0 \\
\text { cat } & 0 & 0 & 0 & 0 \\
\text { goldfish } & 10 & 10 & 0 & 9 \\
\text { shark } & 5 & 20 & 0 & 5 \\
\text { tuna } & 0 & 20 & 0 & 0
\end{array}
$$

A similar calculation gives us that the typicality of goldfish in the concept fish is $\frac{20}{100}$.

The shared contexts of pet and fish are: $e_{\text {fish }}^{\text {pet }}, e_{\text {pet }}^{f i s h}, e_{\text {swim }}^{\text {pet }}, e_{\text {swim }}^{\text {fish }}$ Now, to form combinations of concepts, such as pet fish, the concept is represented in the tensor product space of the two concepts pet and fish. The basic contexts of the concept pet fish are denoted $E^{\text {pet fish }}$ which is the intersection of the set $E^{\text {pet }}$ of the basic contexts of pet, and the set $E^{f i s h}$ of the basic contexts of fish. The assumption is made for the example that $E^{\text {pet fish }}=E_{\text {fish }}^{\text {pet }} E_{\text {pet }}^{\text {fish }}$, where $E_{\text {fish }}^{\text {pet }}=\left\{u \in E^{\text {pet }} \mid u\right.$ is more specific than $\left.e_{\text {fish }}^{\text {pet }}\right\}$, i.e. it is the set of basic contexts of the concept pet that are more specific than the pet is a fish. Note that in tables 7 and 8 , the cardinality of that set is 15 .

The concept pet fish is modelled as

$$
\mid \text { pet fish }\rangle=\sum_{u \in E^{\text {pet fish }}} \frac{1}{\sqrt{15}}|u\rangle \otimes|u\rangle
$$

To look at the membership of goldfish in pet fish, we consider the context the pet is a goldfish, modelled as:

$$
G=P_{\text {goldfish }}^{\text {pet }} \otimes 1^{\text {fish }}=\sum_{u \in E_{\text {goldfish }}^{\text {pet }}}|u\rangle\langle u| \otimes \mathbb{I}
$$

Then the typicality of goldfish in pet fish is given by:

$$
\begin{aligned}
\mu(\text { goldfish }, \text { goldfish, pet fish }) & =\langle\text { pet fish }|G| \text { pet fish }\rangle \\
& =\frac{1}{15} \sum_{v \in E^{\text {pet fish }}}\left\langle v\left|\otimes\left\langle v\left|\sum_{u \in E_{\text {goldfish }}^{\text {pet }}}\right| u\right\rangle\left\langle u\left|\otimes \mathbb{I} \sum_{w \in E^{\text {pet fish }}}\right| w\right\rangle \otimes\right| w\right\rangle \\
& =\frac{1}{15} \sum_{v \in E^{\text {pet fish }}} \sum_{u \in E_{\text {goldfish }}^{\text {pet }}} \sum_{w \in E^{\text {pet fish }}}\langle v \mid u\rangle\langle u \mid v\rangle\langle v \mid w\rangle=\frac{10}{15}
\end{aligned}
$$

We can see that the typicality of goldfish in pet fish at $\frac{2}{3}$ is much higher than the typicality of goldfish in either pet $\left(\frac{1}{10}\right)$ or fish $\left(\frac{2}{10}\right)$.

Moreover, although we have only applied the context goldfish to the pet subsystem, the fish subsystem 
has changed as well:

$$
\begin{aligned}
\nu(\text { goldfish }, \text { pet fish }) & =\frac{G \mid \text { petfish }\rangle}{\sqrt{\langle\text { pet fish }|G| \text { pet fish }\rangle}}=\frac{\sqrt{15}}{\sqrt{10}} \sum_{u \in E_{\text {goldfish }}^{\text {pet }}}|u\rangle\left\langle u\left|\otimes \mathbb{I} \sum_{w \in E^{\text {pet fish }}} \frac{1}{\sqrt{15}}\right| w\right\rangle \otimes|w\rangle \\
& =\frac{1}{\sqrt{10}} \sum_{u \in E_{\text {goldfish }}^{\text {pet }}} \sum_{w \in E^{\text {pet fish }}}|u\rangle\langle u \mid w\rangle \otimes|w\rangle=\frac{1}{\sqrt{10}} \sum_{u \in E_{\text {goldfish }}^{\text {pet }}}|u\rangle \otimes|u\rangle
\end{aligned}
$$

If we constructed a similar context saying that the fish is a goldfish, the concept would collapse to the same state. In the pet fish concept, saying that the pet is a goldfish has the same effect as saying that the fish is a goldfish - and this is the desired outcome. In contrast, if the concept pet fish was modelled without entanglement, for example as:

$$
\begin{aligned}
\left.\mid \text { pet } f i s h^{\prime}\right\rangle & \left.=\mid \text { pet }_{\text {fish }}\right\rangle \otimes\left|f i s h_{\text {pet }}\right\rangle \\
& =\sum_{u \in E_{\text {fish }}^{\text {pet }}} \sum_{v \in E_{\text {pet }}^{f i s h}}|u\rangle \otimes|v\rangle
\end{aligned}
$$

Then

$$
\begin{aligned}
\left.G \mid \text { pet fish }{ }^{\prime}\right\rangle & =\sum_{u \in E_{\text {got dfsh }}^{\text {pet }}}|u\rangle\left\langle u\left|\otimes \mathbb{I} \sum_{v \in E_{\text {fish }}^{\text {pet }}} \sum_{w \in E_{\text {pet }}^{\text {fish }}}\right| v\right\rangle \otimes|w\rangle \\
& =\sum_{u \in E_{\text {goldfish }}^{\text {pet }}}|u\rangle \otimes \sum_{w \in E_{\text {pet }}^{\text {fish }}}|w\rangle
\end{aligned}
$$

where although the pet subsystem has changed to a goldfish, the fish subsystem has not. If we constructed a similar context saying that the fish was a goldfish, we would not obtain the same state. We would instead have a state consisting of a generic pet, and a goldfish. As such, the entanglement is essential to correctly model this problem.

Aerts and Gabora (2005b) go on to describe that this approach could be extended to model the composition of concepts in more complicated structures, up to the sentence level.

Whilst the approach given in Aerts and Gabora (2005b) is able to model the phenomenon of overextension, it suffers for the problem that the space in which concepts must be modelled gets longer dependent on the phrase or sentence length. In Coecke et al. (2010) an approach to modelling language was proposed that combines the very successful distributional models of meaning with the compositional approaches from formal semantics. In brief, this approach models words in differing vector spaces based on their grammatical type. Nouns are modelled in a vector space $N$ for nouns, and sentences are modelled in a vector space $S$ for sentences. Adjectives are modelled as linear maps on the noun space, i.e. matrices in $N \otimes N$, and transitive verbs are bilinear maps from two copies of the noun space to the sentence space, or tensors in $N \otimes S \otimes N$. The benefit of this approach is that arbitrarily long sentences can all be mapped into the same vector space $S$. Whilst this approach was originally developed with an eye to natural language processing, it has also been used to tackle problems in cognitive science.

In Coecke and Lewis (2015), the compositional distributional model is used to examine the pet fish problem, and more generally the problem of overextension. The first approach to tackling the pet fish problem is to view the word pet as an adjective, rather than a noun. Then the parameters of the matrix can be tuned so that the similarity of the vector goldfish to pet fish is greater than the similarity to either pet or fish. The sentence can further be expanded out to look at the conjunctions pet which is a fish and fish which is a pet, and this format is used to model the importance of attributes in the conjunction of concepts. In Hampton (1987), data is collected on the importance of attributes in concepts and their combination. Concepts are considered in pairs that are related to some degree, for example 'Pets', and 'Birds'. Six pairs are considered in total. Participants are asked to generate attributes for each concept and for their conjunctions, where conjunction in this case is rendered as 'Pets which are also Birds', or 'Birds 
which are also Pets'. For example, attributes such as: 'lives in the house', 'is an animal', 'has two legs', are generated for 'Pets', 'Birds'. For each pair of concepts and their conjunction, attributes that had been generated by at least 3 out of the 10 participants were collated. Participants were then asked to rate the importance of each attribute to each concept and to each conjunction. Importance ratings were made on a 7 point verbal scale ranging from 'Necessarily true of all examples of the concept' to 'Necessarily false of all examples of the concept'. Numerical ratings were subsequently imposed ranging from 4 to -2 respectively.

The question then arises of how the importance of attributes in the conjunction of the concepts is related to the importance of attributes in the constituent concepts. Key phenomena are that conjunction is not commutative, that inheritance failure can occur (i.e., an attribute that is important in one of the concepts is not transferred to the conjunction), that attribute emergence can occur, where an attribute that is important in neither of the conjuncts becomes important in the conjunction, and further, that necessity and impossibility are preserved. In order to model this data, Hampton uses a multilinear regression.

In Coecke and Lewis (2015), the phrase $A$ which is a $B$ is modelled as

$$
\mid A \text { which is a } B\rangle=|A\rangle \odot \underline{i s}|B\rangle
$$

where $\underline{i s}$ is a matrix modelling the word $i s$ as a transitive verb.

To model the data from Hampton (1987), parameters for the verb is are found using a numerical solver. Results are that it is essentially possible to model the data exactly. We should note that since a large matrix is used, the numbers of parameters are very large, however as a demonstration of how the compositional model can be applied to psychological data it is still useful.

\subsection{Modelling of over and under-extension in psychological data}

The phenomenon of over and under extension in human concept combination has been extensively investigated in Hampton (1988a,b). In Hampton (1988a), Hampton looks at how the membership of exemplars in conjunctions of concepts compares that the membership of exemplars in the individual concepts. In Hampton (1988b), he examines how the membership of exemplars in disjunctions of concepts compares to that in the individual concepts. The overall findings are that in the conjunctive case, overextension is often seen, and in the disjunctive case, underextension is often seen. Moreover, membership in the conjunction is dependent on the order in which the conjunction is made. As we saw in section 3.2, if concepts are modelled as regions of a feature space, the order of conjunction should not matter.

These phenomena are given a quantum modelling in Aerts (2009). The model is based on a Fock space. The combination of two concepts are considered in two differing ways. Concepts $A$ and $B$ are modelled as states $|A\rangle$ and $|B\rangle$ of a Hilbert space $\mathcal{H}$. Firstly, the disjunction of two concepts $A$ and $B$ are considered as the superposition of their two states: $A$ or $B=\frac{1}{\sqrt{2}}(|A\rangle+|B\rangle)$. The membership $\mu_{A}$ or $B(X)$ of an item $X$ in the concept $A$ or $B$ is given by an orthogonal projection $M_{X}$, i.e.

$$
\begin{aligned}
\mu_{A \text { or } B}(X) & =\frac{1}{2}\left(\left\langleA|+\langle B|) M_{X}(|A\rangle+|B\rangle)\right.\right. \\
& =\frac{1}{2}\left(\left\langle A\left|M_{X}\right| A\right\rangle+\left\langle B\left|M_{X}\right| B\right\rangle+\operatorname{Re}\left(\left\langle A\left|M_{X}\right| B\right\rangle\right) \quad=\frac{1}{2}\left(\mu_{A}(X)+\mu_{B}(X)\right)+\operatorname{Re}\left(\left\langle A\left|M_{X}\right| B\right\rangle\right)\right.
\end{aligned}
$$

where this last term is the interference term, as in section 3.4.

On the other hand, the disjunction of the two concepts can be modelled in two copies of $\mathcal{H}$, as $|A\rangle \otimes|B\rangle$. The operator $M_{X} \otimes M_{X}$ is a projection operator over the space that asks whether $X$ is a member of $A$ and whether $X$ is a member of $B$. To determine the membership of $X$ in $A$ or $B$, we therefore calculate

$$
\begin{aligned}
\mu_{A} \text { or } B(X) & =1-\left(\left\langleA|\otimes\langle B|)\left(\mathbb{I}-M_{X}\right) \otimes\left(\mathbb{I}-M_{X}\right)(|A\rangle \otimes|B\rangle)\right.\right. \\
& =1-\left(1-\mu_{A}(X)\right)\left(1-\mu_{B}(X)\right)=\mu_{A}(X)+\mu_{B}(X)-\mu_{A}(X) \mu_{B}(X)
\end{aligned}
$$

which gives us a classical-style disjunction. 
For the case of conjunction, a classical-style conjunction can be modelled in $\mathcal{H} \otimes \mathcal{H}$ simply by taking

$$
\begin{aligned}
\mu_{A} \text { and } B(X) & =\left\langle A|\otimes\langle B|)\left(M_{X} \otimes M_{X}\right)(|A\rangle \otimes|B\rangle)\right. \\
& =\mu_{A}(X) \mu_{B}(X)
\end{aligned}
$$

and the conjunction in one copy of $\mathcal{H}$ is modelled as $\frac{1}{\sqrt{2}}(|A\rangle+|B\rangle)$ again. This means that in the single space, conjunction and disjunction are the same.

The single space and the double space are combined in a Fock space $\mathcal{H} \otimes \mathcal{H} \oplus \mathcal{H}$. The concept overall is represented as

$$
\psi(A, B)=m e^{i \theta}|A\rangle \otimes|B\rangle+\frac{n e^{i \phi}}{\sqrt{2}}(|A\rangle+|B\rangle)
$$

where $m^{2}+n^{2}=1$. Then,

$$
\begin{aligned}
& \mu_{A \text { and } B}(X)= \\
& \qquad\left(m e ^ { i \theta } \langle A | \otimes \left\langleB\left|+\frac{n e^{i \phi}}{\sqrt{2}}(\langle A|+\langle B|)\right) M_{X} \otimes M_{X} \oplus M_{X}\left(m e^{i \theta}|A\rangle \otimes|B\rangle+\frac{n e^{i \phi}}{\sqrt{2}}(|A\rangle+|B\rangle)\right)\right.\right. \\
& =m^{2} \mu_{A}(X) \mu_{B}(X)+n^{2}\left(\frac{1}{2}\left(\mu_{A}(X)+\mu_{B}(X)\right)+\operatorname{Re}\left(\left\langle A\left|M_{X}\right| B\right\rangle\right)\right)
\end{aligned}
$$

A similar calculation gives us that

$$
\mu_{A \text { or } B}(X)=m^{2}\left(\mu_{A}(X)+\mu_{B}(X)-\mu_{A}(X) \mu_{B}(X)\right)+n^{2}\left(\frac{1}{2}\left(\mu_{A}(X)+\mu_{B}(X)\right)+\operatorname{Re}\left(\left\langle A\left|M_{X}\right| B\right\rangle\right)\right)
$$

Essentially, the part of the concept that is modelled as a superposition contributes to the combinations $A$ and $B$ or $A$ or $B$ to move the combination away from a classical combination of concepts.

Aerts (2009) then shows that the data from Hampton $(1988 \mathrm{a}, \mathrm{b})$ can be modelled in a space $\mathbb{R}^{2} \otimes \mathbb{R}^{2} \oplus \mathbb{R}^{4}$, in which the angles $\theta$ and $\phi$ are tuned.

In Sozzo (2014) the same sort of Fock space modelling is used to describe problematic borderline cases in negation. Sozzo (2014) models the data from Alxatib and Pelletier (2011) by modelling the concepts tall and not tall as separate states in the one-system part of the space, $\mathcal{H}$. The combinations tall and not tall and neither tall not not tall are then modelled in the two-system part of the space $\mathcal{H} \otimes \mathcal{H}$, and combined with the superposition of $|A\rangle$ and $\left|A^{\prime}\right\rangle$. As such, the superposition of the two states combines with their logical combination to produce the effects seen in Alxatib and Pelletier (2011).

In Sozzo (2015); Aerts et al. (2015) this Fock space modelling of conjunction and negation are combined. If a concept $A$ is modelled by $|A\rangle$, then the negation of that concept is modelled by another state $\left|A^{\prime}\right\rangle$. Within the first part of the Fock space, in which the concepts $A$ and $B$ are modelled within a single Hilbert space $\mathcal{H}$, negations are modelled using $\left|A^{\prime}\right\rangle$ and $|A B\rangle$. However, within the two-system part of the Fock space, the negations of concepts can be represented classically, as $A$ and $B$ or $A$ or $B$ are. Specifically, the membership of $X$ in the negation of $A$ is modelled as:

$$
\left\langle A\left|\mathbb{I}-M_{X}\right| A\right\rangle=1-\mu_{A}(X)
$$

This system is able to successfully model data from Hampton (1997) and from Sozzo (2015).

\subsubsection{Ambiguity, entanglement, and concept combination}

A key part of human concept use is how we express these concepts in language. However, when concepts are expressed in language, the language can have one word which refers to the same concept. A well-used examples is the word bank. If I say 'I'm going to the bank to deposit some money', then bank has a different meaning than if I say 'I'm going to the bank to fish'. In Bruza et al. (2012), an experiment is performed that looks into pairs of such ambiguous words and their combinations. An example is apple chip. This could 
mean a part of a Macintosh computer, or a slice of dried fruit. If I interpret apple in the sense computer, then it is very unlikely that I should interpret chip as a slice, or part, or the computer. Similarly, if I interpret apple in the sense fruit, then it is very unlikely that I will interpret chip in the sense of a computer chip.

A quantum modelling of this phenomenon would seem very appropriate: if we view each individual concept as modelled in a Hilbert space $\mathcal{H}$, and I disambiguate the meanings so that I have 4 vectors $\mid$ apple $\left._{\text {computer }}\right\rangle, \mid$ apple $\left._{\text {fruit }}\right\rangle, \mid$ chip $\left._{\text {computer }}\right\rangle, \mid$ chip $\left._{\text {slice }}\right\rangle$, then we can model the combined concept in $\mathcal{H} \otimes \mathcal{H}$ as:

$$
\text { apple chip }=\frac{\left.\left.\left.\left.\mid \text { apple }_{\text {computer }}\right\rangle \mid \text { chip }_{\text {computer }}\right\rangle+\mid \text { apple }_{\text {fruit }}\right\rangle \mid \text { chip }_{\text {fruit }}\right\rangle}{\sqrt{2}}
$$

Given a state $|p s i\rangle$ that the agent is in, the interpretation of the combination will collapse to either the computer sense or the fruit sense.

However, another form of combination is as follows. We could model apple as $\mid$ apple $\rangle=\frac{1}{\sqrt{2}} \mid$ apple $\left._{\text {computer }}\right\rangle+$

$$
\begin{aligned}
& \left.\left.\left.\left.\mid \text { apple }_{\text {fruit }}\right\rangle \text {, and } \mid \text { chip }\right\rangle=\frac{1}{\sqrt{2}} \mid \text { chip }_{\text {computer }}\right\rangle+\mid \text { chip }_{\text {slice }}\right\rangle \\
& \text { apple chip }=\mid \text { apple }\rangle \otimes \mid \text { chip }\rangle \\
& \left.\left.\left.=\frac{1}{2}\left(\mid \text { apple }_{\text {computer }}\right\rangle \mid \text { chip }_{\text {computer }}\right\rangle+\mid \text { apple }_{\text {computer }}\right\rangle \mid \text { chip }_{\text {fruit }}\right\rangle \\
& \left.\left.\left.\left.\left.+\mid \text { apple }_{\text {fruit }}\right\rangle \mid \text { chip }_{\text {computer }}\right\rangle+\mid \text { apple }_{\text {fruit }}\right\rangle \mid \text { chip }_{\text {fruit }}\right\rangle\right)
\end{aligned}
$$

In this case, a participant could interpret the combination apple chip in any of the four possible combinations.

This kind of phenomenon is investigated in Bruza et al. (2015) In these experiments, the participant is first primed for one of the senses of the combination - for example, the word banana is shown to them before they are asked to make a decision on the interpretation of the compound. The participants are then asked to describe the interpretation they have made. These interpretations are used to build a set of probabilities for the interpretations of the compounds, and these probabilities are analyzed to determine whether the compound is separable or entangled. There are a number of factors which need to be accounted for in this, which are discussed in Dzhafarov and Kujala (2016) and which we discussed in section 3.7.

The results are that in general, there is no clear evidence for the entanglement of these concepts in the human mental lexicon. However, in a number of cases, there is some evidence of phenomena occurring which are not completely explained by a classical probabilistic model.

Another approach to modelling the ambiguity of words is to model words as density matrices (Piedeleu et al., 2015; Meyer and Lewis, 2020). This was proposed as an extension of the compositional distributional model of Coecke et al. (2010). As mentioned in 3.1, a density matrix is a probability distribution over a number of quantum states:

$$
\rho=\sum_{i} \alpha_{i}\left|v_{i}\right\rangle\left\langle v_{i}\right|
$$

where $\forall i, \alpha_{i}>0$ and $\sum_{i} \alpha_{i}=1$. The idea of using density matrices to model ambiguity runs as follows. Each sense of a word is modelled as a vector, and then these senses are mixed together following equation (118) above. Crucially, the same compositional framework as in the standard vector based model is available. This means that as words are used in context, their meaning can be disambiguated, in the same way that humans are able to. In Meyer and Lewis (2020), a neural approach to building density matrices for words is proposed and tested on a set of sentence similarity data that requires words to be disambiguated correctly. The representations learnt by Meyer and Lewis (2020) perform very well, outperforming some of the strongest neural network systems for language processing currently available.

\subsection{Cognitive and neuronal structures}

In the foregoing sections, the level of modelling was at a high, cognitive level. There is no attempt to form any match between these models and actual processes in the brain. However, there has been a range of research into using quantum theory to model cognitive processes at a level that is closer to a neuronal level. 
Fuss and Navarro (2013) describe a quantum model of two-alternative forced choice (2AFC) experiments. In this sort of experiment, the participant is asked to make a choice about a stimulus. For example, there may be two shapes on a computer screen and the participant is asked which one is a square. The participant must choose one or the other, and does not have to choice to say neither. Information about the nature of processing is inferred from reaction times. For example, the average reaction time to the image in figure $8 \mathrm{a}$ may be faster than the average reaction time to the image in $8 \mathrm{~b}$
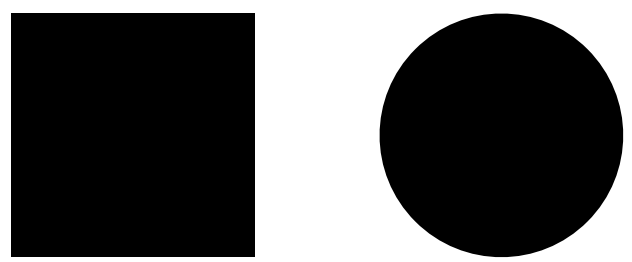

(a) A square and a circle
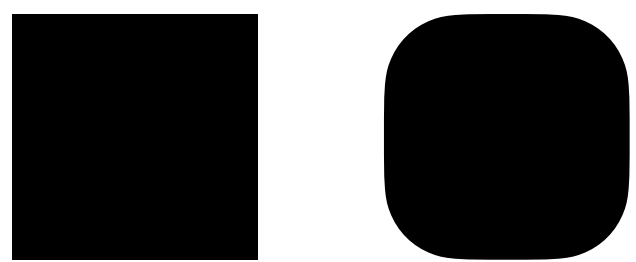

(b) A square and another with rounded edges

Figure 8: Differing stimuli in a 2AFC experiment

A standard way of modelling reaction times in this sort of experiment is to say that a pair of neurons, or groups of neurons is responsible for accumulating evidence for one choice or the other, and that when the evidence hits a threshold, the participant reacts. The two accumulators are in a race to get to the threshold. So in the examples in figure 8 , evidence is being accumulated by one accumulator for left and one for right. In figure 8a, evidence for right would only accumulate slowly, and so the reaction left would happen more quickly, whereas in figure 8b, more of the evidence would be added to the right accumulator, and so overall the threshold would take longer to reach. This is usually modelled as a random walk with drift. An extension to a simple random walk model is to consider that multiple of these random walks could be happening in parallel. However, having multiple random walks happening in parallel can still only produce a model where evidence is accumulated. On the other hand, a model that explicitly models neurons and the connections between them (connectionist models) can account for inhibitory connections, i.e. that evidence for one choice can inhibit the accumulation of evidence for the other choice.

In Fuss and Navarro (2013) a parallel random walk model using quantum theory is proposed. This allows for the kinds of inhibition we just mentioned to be modelled, by means of interference. This kind of model can produce qualitatively different probability distributions over the search space, allowing for more efficient decision making to be carried out. The authors show that this kind of model can fit 2AFC experimental data more closely than a simple standard random walk with drift (although there are more complex models which may do better).

More recently, Khrennikov et al. (2018) have begun to formulate a description of neuronal substrates within quantum terms. This does not claim that neuronal and cognitive activity are being modelled at the quantum level (although models to explain cognitive activity through actual quantum modelling has been proposed in Hameroff (2014); Penrose (1990). Rather, the idea is that quantum theory can be used to represent the information and activity present in neuronal assemblies.

\subsection{Tensor product representations}

Whilst not explicitly quantum inspired, the tensor product representations of Smolensky (1990) use structures which are native to quantum systems. The aim in Smolensky (1990) is to describe a means of representing symbolic structures and their composition within a connectionist substrate. Connectionist networks (nowadays usually called neural networks) are based on the idea that cognitive systems can be modelled by the interconnection of small artificial neurons, and indeed the motivation for this is clear - as our cognitive system is based in the interconnection of many interacting neurons. There are other aspects to the basis of our cognitive system, such as the physical bodies that each of us inhabits, but undeniably our (actual) neural network plays a part in cognition. Also, there is no claim here that artificial neural networks bear any 
resemblance to real neural networks. However, artificial neural networks are extremely successful at various tasks.

An aspect of cognition that humans excel at is symbolic manipulation. This doesn't mean complex mathematics, but the ability that if we understand the sentence Jen is Junpa's mother, we can also understand the sentence Junpa is Jen's mother, even if we judge it to be false.

Artificial neural networks don't have an obvious means of representing symbols so that we can manipulate them in this way, and in Smolensky (1990); Smolensky and Legendre (2006) a method for doing so is proposed.

Vectors and tensor products are used to represent objects, roles, and structures. The underlying idea is that roles, such as subject, or object, are represented as vectors, and fillers, also represented as vectors, can be bound to these roles using the tensor product, and collections of roles and fillers are combined using vector addition. Recursive structures such as trees can be represented. The contents of the leaves are encoded in the fillers, and role vectors encode the tree structure.

To be able to represent trees, we view the binding of a role and a filler as itself a filler, i.e. the filler is itself a complex structure, and not an atomic symbol. The binding $\mathrm{f} / r$ of a filler $\mathrm{f}$ to a role $r$ is represented as a vector $\mathbf{f} \otimes \mathbf{r}$. A sentence $\mathbf{s}$ is represented as a sum of filler/role bindings $\sum_{i} \mathbf{f}_{i} \otimes \mathbf{r}_{i}$, and these can be applied recursively. For example, let $\mathbf{s}=[N P$ VP] be a binary tree with left and right subtrees NP and VP. Let $\mathbf{s}, \mathbf{v}_{1}, \mathbf{v}_{2}$ be the vectors representing $\mathbf{s}, N P, V P$. The connectionist representation of $\mathbf{s}$ is:

$$
\mathbf{s}=\mathbf{v}_{1} \otimes \mathbf{r}_{0}+\mathbf{v}_{2} \otimes \mathbf{r}_{1}
$$

If VP is a tree rather than an atomic symbol, it can be expressed in terms of its left and right subtrees $\mathrm{VP}=[\mathrm{Vt} \mathrm{NP}]$. If $\mathbf{v}_{3}, \mathbf{v}_{4}$ represent the trees Vt, NP, then the structure $\mathbf{s}=[\mathrm{NP}$ [Vt NP]] has the following representation:

$$
\begin{aligned}
\mathbf{s} & =\mathbf{v}_{1} \otimes \mathbf{r}_{0}+\left(\mathbf{v}_{3} \otimes \mathbf{r}_{0}+\mathbf{v}_{4} \otimes \mathbf{r}_{1}\right) \otimes \mathbf{r}_{1} \\
& =\mathbf{v}_{1} \otimes \mathbf{r}_{0}+\mathbf{v}_{3} \otimes\left(\mathbf{r}_{0} \otimes \mathbf{r}_{1}\right)+\mathbf{v}_{4} \otimes\left(\mathbf{r}_{1} \otimes \mathbf{r}_{1}\right) \\
& \equiv \mathbf{v}_{1} \otimes \mathbf{r}_{0}+\mathbf{v}_{3} \otimes \mathbf{r}_{01}+\mathbf{v}_{4} \otimes \mathbf{r}_{11}
\end{aligned}
$$

A notable feature of this representation is that the vector space in which concepts live must be arbitrarily large, depending on the size of the structure to be represented. Symbols at depth $d$ in a binary tree are realized by $\mathcal{S}_{(d)}$, the $F R^{d}$-dimensional vector space formed from vectors of the form $\mathbf{f} \otimes \mathbf{r}_{i} \otimes \mathbf{r}_{j} \otimes \cdots \otimes \mathbf{r}_{k}$ with $d$ role vectors, where $F$ is the dimension of the filler vectors $\mathbf{f}$ and $R$ is the dimension of the individual role vectors $\mathbf{r}_{i}$. A vector space containing all vectors in $\mathcal{S}_{(d)}$ for all $d$ is:

$$
\mathcal{S}^{*} \equiv \mathcal{S}_{(0)} \oplus \mathcal{S}_{(1)} \oplus \mathcal{S}_{(2)} \oplus \cdots
$$

Vectors $\mathbf{s}_{(i)}$ are embedded into this space, meaning that the normal operation of vector addition can be used to combine sentence components.

Once a set of concepts (e.g. Jen, loves, and Junpa) have been combined into one vector representation, it is also possible to pull the representation apart, and recombine it. This is done by means of an unbinding mechanism. Suppose we form the sentence representation

$$
|S\rangle=|J e n\rangle \otimes|s u b j\rangle+\mid \text { runs }\rangle \otimes|v e r b\rangle
$$

we can ask 'who is the subject of the sentence' by unbinding $|s u b j\rangle$ from the sentence. If role vectors are all orthogonal, then the unbinding is simply done by taking the inner product of $|S\rangle$ with the role vector: $\langle S \mid s u b j\rangle=|J e n\rangle$

\begin{tabular}{ccc}
\hline Structure & Symbolic & Connectionist \\
\hline Set & $\mathbf{f}$ & $\mathbf{f} \in V_{F}$ \\
String & $\mathbf{f}_{i} / r_{i}$ & $\mathbf{f}_{i} \otimes \mathbf{r}_{i}$ \\
Tree & $\mathbf{s}=\left\{\mathbf{f}_{i} / r_{i}\right\}$ & $\mathbf{s}=\sum_{i} \mathbf{f}_{i} \otimes \mathbf{r}_{i} \in \mathcal{S}^{*}$ \\
\hline
\end{tabular}

Table 9: Space of descriptions in ICS 
Tensor product networks have recently been used extensively to examine the structure of recurrent neural networks (McCoy et al., 2019), and also as a potential substrate for quantum language processing (Wiebe et al., 2019). Moreover, very similar structures have been used in the neural-based computing framework Nengo (Bekolay et al., 2014). These latter structures are different in that they use circular convolution (Plate, 1995) rather than tensor product to bind roles and fillers. The benefit of using circular convolution rather than the tensor product is that all vectors are mapped into one shared space. An approximate unbinding mechanism can be employed by using circular correlation with a query vector.

Tensor product representations have also been used in Pothos and Trueblood (2015) to model how the similarity of objects can depend on where the similarity is to be found. For example, given two birds with a spotted pattern, if the spotted pattern is found on the wings of one bird and the back of the other, they will not be judged as similar as if they both have the spotted patter on their wings. The use of role vectors in the tensor product representations of Smolensky (1990); Smolensky and Legendre (2006) is leveraged to account for this phenomenon.

The compositional distributional model Coecke et al. (2010), mentioned in section 3.8 has the property that all representations are mapped into one shared space, and shares some structures similar to the ICS model. In Al-Mehairi et al. (2017) it is shown that the ICS model can be mapped on to the compositional distributional model, to form a categorical compositional model of cognition.

A further mapping from ICS to the compositional distributional model can be considered. One of the most useful aspects of the compositional distributional model is the way that functional words such as adjectives and verbs modify the nouns they are applied to, either by mapping them to another vector in the noun space, or by mapping them into a sentence space. Within the ICS model, this does not happen in the same way. Consider the following problem, as investigated in Martin and Doumas (2020). Under the assumption that the concepts we use are expressed by the language we use, the way that we compose concepts can be examined by looking at properties of words and phrases. Within the field of neural modelling, a key question is how neural representations of two concepts can be composed into a single combined representation. Neural representations of concepts are commonly modelled as (normalized) vectors, and similarity between concept representations calculated by the inner product. Two common proposals for how to combine neural representations are via firstly tensor product binding or holographic reduced representations, as described above, or secondly vector addition (Hummel, 2011).

The problem with tensor product binding is that it does not transform the argument of a relation in the necessary way. Consider vectors $|f l u f f y\rangle,|c a t\rangle,|d o g\rangle$. If the combinations fluffy $\operatorname{dog}$ and fluffy cat are represented by the tensor product of the two component vectors:

$$
\begin{aligned}
\text { fluffy dog } & =\mid \text { fluffy }\rangle \otimes \mid \text { dog }\rangle \\
\text { fluffy cat } & =\mid \text { fluffy }\rangle \otimes \mid \text { cat }\rangle
\end{aligned}
$$

then the similarity between fluffy cat and fluffy dog is just the similarity between cat and dog, since

$$
\begin{aligned}
& (\langle f l u f f y| \otimes\langle\text { dog }|)(|f l u f f y\rangle \otimes|c a t\rangle) \\
& \quad=\langle\text { fluffy }| \text { fluffy }\rangle \otimes\langle\text { dog }| \text { cat }\rangle \\
& =\langle\text { dog }| \text { cat }\rangle
\end{aligned}
$$

This is undesirable, as can be see in figure 9 .

On the other hand, vector addition has been shown to be successful in modelling concept combination Hummel (2011); Martin and Doumas (2020), and has a clear neural correlate, termed synchronous binding. However it is arguably not able to express gradations in meaning that are needed in terms like red car vs red wine. For example, given $|r e d\rangle$, $\mid$ wine $\rangle,|c a r\rangle$, suppose that wine and car are not very similar, say $\langle$ wine $|$ car $\rangle=0.1$, and they are each fairly similar to red, say $\langle$ red $|$ car $\rangle=\langle$ red $|$ wine $\rangle=0.5$. Then, adding 


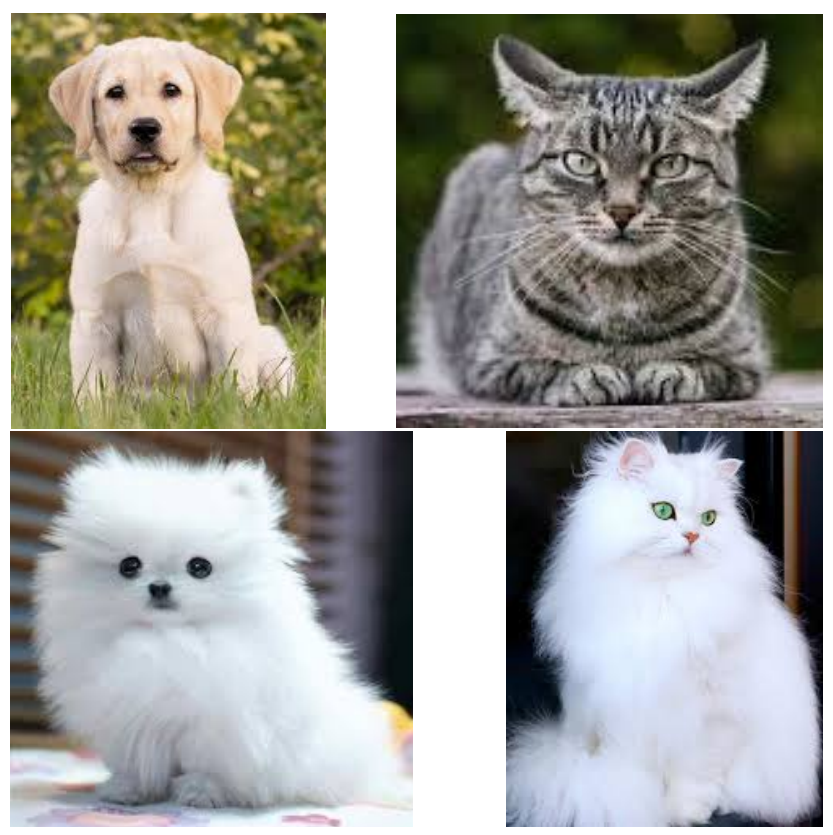

Figure 9: Fluffy cats and fluffy dogs are more similar than cats and dogs

vectors and normalizing:

$$
\begin{aligned}
\text { red car } & =\frac{\mid \text { red }\rangle+\mid \text { car }\rangle}{\sqrt{3}} \\
\text { red wine } & =\frac{\mid \text { red }\rangle+\mid \text { wine }\rangle}{\sqrt{3}}
\end{aligned}
$$

and

$$
\begin{aligned}
&\langle\text { red car }| \text { red wine }\rangle \\
&=\left.\left.\frac{1}{3}(\langle\text { red }| \text { red }\rangle+\langle\text { red }| \text { car }\right\rangle+\langle\text { red }| \text { wine }\right\rangle \\
&+\langle\text { wine }| \text { car }\rangle) \\
&= \frac{2}{3}+\frac{\langle\text { wine }| \text { car }\rangle}{3}
\end{aligned}
$$

i.e., the similarity has increased from 0.1 to just over two-thirds. However, this again does not accord with our intuitions: the similarity between wine and car should not increase dramatically when the adjective red is applied, at least in part because applying this adjective does not even make the colours very similar. It might be argued that this can be overcome by downweighting the importance of the adjective in an adjective-noun combination. However, there are situations when we would like similarity to increase when the adjective is applied: the adjective red in red car and red van should increase similarity.

A approach than can address both these aspects is the quantum-inspired approach of Coecke et al. (2010). Since adjectives and verbs are represented as matrices rather than vectors, the combination of an adjective and a verb transforms the argument so that fluffy cats can be moved closer to fluffy dogs, i.e. the problem in equation (126) is not encountered. On the other hand, the application of an adjective like red does not force red car to become similar to red wine. How? Essentially,the use of a matrix gives enough 'space' for different uses of words to be represented. 


\subsubsection{Reasoning in vector spaces}

Another line of work looks at using vector space methods to model structure in concepts. A key characteristic of the concepts we use is that they are hierarchical. As well as categorizing individual entities, we want to talk about types of entities - for example, we want to simply say that tigers are dangerous, rather than 'that tiger is dangerous. And that tiger is dangerous. And that one. And...'. Within a quantum model, we can start doing this by considering concepts as subspaces of a vector space, and using the lattice structure of the subspaces, or equivalently their projectors, to reason with. This was originally proposed by Birkhoff and Von Neumann (1936)

The density matrix variant of the compositional distributional model of Coecke et al. (2010) forms an extension of the quantum logic formed by the lattice of projectors. Words are modelled as positive operators, and hierarchy in concepts is modelled via the Löwner order. In Bankova et al. (2019); Sadrzadeh et al. (2018) it is shown that the Löwner order is compatible with the compositional model used. In Lewis (2019) a concrete modelling of words as positive operators is given which is shown to model human judgements of entailment very well. Further work into modelling negation is underway Lewis (2020), and work on modelling other forms of logical structure such as generalized quantifiers has also been undertaken by Hedges and Sadrzadeh (2019); Dostál et al. (2021). Another approach to modelling negation in vector space representations of meaning is seen in Widdows and Peters (2003), this time within an information retrieval setting.

\section{Discussion}

There are clearly a number of applications of quantum theory to problems in cognitive science. We have seen that human use of concepts has some unusual features, which seem to be well modelled using a quantum framework.

In the approach proposed by Pothos et al. (2013); Busemeyer et al. (2011); Wang et al. (2013) and used in numerous other papers, a concept is modelled as a subspace of a Hilbert space, and the knowledge state of an agent is modelled as a state in the Hilbert space. The theory therefore crucially incorporates the way in which decisions are dependent on the state of the agent, and how the presence or absence of information and the order in which stimuli occur can affect judgements. Within this model, the phenomenon of interference does a lot of work. In the modelling of the asymmetry of similarity judgements, $\| P_{\text {China }}\left|\psi_{\text {Korea }}\right\rangle\left\|^{2} \cdot\right\| P_{\text {Korea }}|\psi\rangle \|^{2}$ is interpreted as the similarity of Korea to China. However, in the model of the conjunction fallacy, this is interpreted as the conjunction of $A$ and then $B$, as it also is in the question order model.

The conjunction and negation model proposed by Aerts and Gabora (2005b) also models concepts as subspaces of a vector space. Here, the subspaces have a very high dimensionality and are based on very specific contexts that objects are seen in. The notion of entanglement plays a key part in modelling the combination of concepts such as pet fish. Aerts (2009); Sozzo (2015); Aerts et al. (2015) model concepts as states within a vector space, and again use the notion of entanglement. In this latter work, the vector spaces used are smaller - for example around 8 dimensional. A drawback of these compositional methods is that in all cases, the size of the underlying space grows as more concepts are added to the combination. This has at least two drawbacks: for more complex concepts, spaces may grow unfeasible large, and furthermore it means that combinations of unequal numbers of concepts are not easy to compare.

Various papers have examined whether a form of contextuality is present in human concept use. In some cases, a claim of contextuality is erroneous, as argued by Dzhafarov and Kujala (2016). On the other hand, Dzhafarov (2019); Basieva et al. (2019) have shown that experiments can be designed in which human decision making does show contextuality. If human decision making does indeed display true contextuality, then the use of quantum computing to model these forms of decision may provide a very useful substrate. However, in many cases there is no evidence of contextuality over and above the effect of direct influences, a more straightforward form of context-dependence.

Vector-based models of meaning and compositionality have been extremely successful in modelling language (Coecke et al., 2010; Sadrzadeh et al., 2018; Kartsaklis and Sadrzadeh, 2016; Smolensky, 1990; McCoy et al., 2019). These models use the same fundamental structures as quantum theory, and extensions of the 
compositional distributional mode have incorporated crucially quantum phenomena such as density matrices. The models proposed in Smolensky (1990) can be argued to have relevance for realistic neuronal modelling Bekolay et al. (2014). Moreover, theory from Smolensky (1990) has been developed for potential implementation on quantum computers in Wiebe et al. (2019), and the model from Coecke et al. (2010) has actually been implemented on a modern quantum computer (Lorenz et al., 2021).

\subsection{Potential for quantum computing to contribute to cognitive science}

So what is the potential for cognitive science within the realm of quantum computing? On the one hand, we can simply say that human cognitive faculties are not computers, whether classical or logical. However, this is unnecessarily negative. There are a wide range of cognitive processes that are well modelled using quantum theory. Models of similarity, judgement, and decision making moreover seem to be well modelled even in fairly low-dimensional spaces.

More complex models, that incorporate compositionality at a more complex level, have been implemented on quantum computers, and the potential for the benefits of this are large. Drawing together some of the theory from the similarity and decision making models to form a unified whole with models of language is an important way forward for this endeavour. One way to progress this would be to consider a form of compositionality that can be applied at the cognitive, rather than the language level, like a language of thought Fodor (1975). Using such a compositional model together with the right sort of representations and notions of similarity and decision making has the potential to form a very powerful model of cognition, which can be implemented natively on computers that in the future may be hugely more powerful than classical computers.

\section{Conclusions}

In this chapter we have covered a range of quantum approaches to cognitive tasks. These approaches all have the capacity to model certain aspects of human cognitive processes more faithfully than other approaches. The compositional models of Smolensky (1990); Coecke et al. (2010) may be able to extend the purely cognitive approaches to model more complex phenomena in an intrinsically quantum way. Moreover, these compositional models are already being implemented on quantum computers. Hence, using quantum computing for cognitive simulation, and AI more generally, has a bright future ahead of it.

\section{References}

Aerts, D. (2009). Quantum structure in cognition. Journal of Mathematical Psychology, 53(5):314-348.

Aerts, D. and Gabora, L. (2005a). A theory of concepts and their combinations I: The structure of the sets of contexts and properties. Kybernetes, 34(1/2):167-191.

Aerts, D. and Gabora, L. (2005b). A theory of concepts and their combinations II: A Hilbert space representation. Kybernetes, 34(1/2):192-221.

Aerts, D., Gabora, L., and Sozzo, S. (2013). Concepts and Their Dynamics: A Quantum-Theoretic Modeling of Human Thought. Topics in Cognitive Science, 5(4):737-772.

Aerts, D., Sozzo, S., and Veloz, T. (2015). Quantum structure of negation and conjunction in human thought. Frontiers in Psychology, 6.

Al-Mehairi, Y., Coecke, B., and Lewis, M. (2017). Categorical Compositional Cognition. In de Barros, J. A., Coecke, B., and Pothos, E., editors, Quantum Interaction, Lecture Notes in Computer Science, pages 122-134, Cham. Springer International Publishing. 
Alxatib, S. and Pelletier, F. J. (2011). The Psychology of Vagueness: Borderline Cases and Contradictions. Mind Es Language, 26(3):287-326.

Ashby, F. G. and Gott, R. E. (1988). Decision rules in the perception and categorization of multidimensional stimuli. Journal of Experimental Psychology: Learning, Memory, and Cognition, 14(1):33-53.

Bankova, D., Coecke, B., Lewis, M., and Marsden, D. (2019). Graded hyponymy for compositional distributional semantics. Journal of Language Modelling, 6(2):225.

Basieva, I., Cervantes, V. H., Dzhafarov, E. N., and Khrennikov, A. (2019). True contextuality beats direct influences in human decision making. Journal of Experimental Psychology: General, 148(11):1925-1937.

Bekolay, T., Bergstra, J., Hunsberger, E., DeWolf, T., Stewart, T. C., Rasmussen, D., Choo, X., Voelker, A., and Eliasmith, C. (2014). Nengo: A Python tool for building large-scale functional brain models. Frontiers in Neuroinformatics, 7.

Birkhoff, G. and Von Neumann, J. (1936). The Logic of Quantum Mechanics. Annals of Mathematics, $37(4): 823-843$.

Bruza, P., Busemeyer, J. R., and Gabora, L. (2009). Introduction to the special issue on quantum cognition. Journal of Mathematical Psychology, 53(5):303-305.

Bruza, P. D., Kitto, K., Ramm, B., Sitbon, L., Song, D., and Blomberg, S. (2012). Quantum-like nonseparability of concept combinations, emergent associates and abduction. Logic Journal of the IGPL, 20(2):445-457.

Bruza, P. D., Kitto, K., Ramm, B. J., and Sitbon, L. (2015). A probabilistic framework for analysing the compositionality of conceptual combinations. Journal of Mathematical Psychology, 67:26-38.

Busemeyer, J. R., Pothos, E. M., Franco, R., and Trueblood, J. S. (2011). A quantum theoretical explanation for probability judgment errors. Psychological Review, 118(2):193-218.

Cervantes, V. H. and Dzhafarov, E. N. (2019). True contextuality in a psychophysical experiment. Journal of Mathematical Psychology, 91:119-127.

Coecke, B. and Lewis, M. (2015). A Compositional Explanation of the Pet Fish Phenomenon. arXiv:1509.06594 [cs, math].

Coecke, B., Sadrzadeh, M., and Clark, S. (2010). Mathematical Foundations for a Compositional Distributional Model of Meaning. arXiv:1003.4394 [cs, math].

Dostál, M., Sadrzadeh, M., and Wijnholds, G. (2021). Fuzzy Generalised Quantifiers for Natural Language in Categorical Compositional Distributional Semantics. In Mojtahedi, M., Rahman, S., and Zarepour, M. S., editors, Mathematics, Logic, and Their Philosophies: Essays in Honour of Mohammad Ardeshir, Logic, Epistemology, and the Unity of Science, pages 135-160. Springer International Publishing, Cham.

Dzhafarov, E. N. (2019). Contextuality and probability in quantum mechanics and beyond: A preface. Philosophical Transactions of the Royal Society A: Mathematical, Physical and Engineering Sciences, 377(2157):20190371.

Dzhafarov, E. N. and Kujala, J. V. (2014). On Selective Influences, Marginal Selectivity, and Bell/CHSH Inequalities. Topics in Cognitive Science, 6(1):121-128.

Dzhafarov, E. N. and Kujala, J. V. (2016). Context-content systems of random variables: The Contextualityby-Default theory. Journal of Mathematical Psychology, page 23.

Fodor, J. A. (1975). The Language of Thought. Harvard University Press. 
Franco, R. (2009). The conjunction fallacy and interference effects. Journal of Mathematical Psychology, 53(5):415-422.

Fuss, I. G. and Navarro, D. J. (2013). Open Parallel Cooperative and Competitive Decision Processes: A Potential Provenance for Quantum Probability Decision Models. Topics in Cognitive Science, pages $\mathrm{n} / \mathrm{a}-\mathrm{n} / \mathrm{a}$.

Gayler, R. W. (2003). Vector Symbolic Architectures Answer Jackendoff's Challenges for Cognitive Neuroscience. In Joint International Conference on Cognitive Science, page 6.

Hameroff, S. (2014). Quantum Walks in Brain Microtubules-A Biomolecular Basis for Quantum Cognition? Topics in Cognitive Science, 6(1):91-97.

Hampton, J. A. (1987). Inheritance of attributes in natural concept conjunctions. Memory $\&$ Cognition, $15(1): 55-71$.

Hampton, J. A. (1988a). Disjunction of natural concepts. Memory \& Cognition, 16(6):579-591.

Hampton, J. A. (1988b). Overextension of conjunctive concepts: Evidence for a unitary model of concept typicality and class inclusion. Journal of Experimental Psychology: Learning, Memory, and Cognition, $14(1): 12-32$.

Hampton, J. A. (1997). Conceptual combination: Conjunction and negation of natural concepts. Memory \& Cognition, 25(6):888-909.

Hedges, J. and Sadrzadeh, M. (2019). A generalised quantifier theory of natural language in categorical compositional distributional semantics with bialgebras. Mathematical Structures in Computer Science, $29(6): 783-809$.

Hummel, J. E. (2011). Getting symbols out of a neural architecture. Connection Science, 23(2):109-118.

Kartsaklis, D. and Sadrzadeh, M. (2016). Distributional Inclusion Hypothesis for Tensor-based Composition. In Proceedings of COLING 2016, the 26th International Conference on Computational Linguistics: Technical Papers, pages 2849-2860, Osaka, Japan. The COLING 2016 Organizing Committee.

Khrennikov, A., Basieva, I., Dzhafarov, E. N., and Busemeyer, J. R. (2014). Quantum Models for Psychological Measurements: An Unsolved Problem. PLoS ONE, 9(10):e110909.

Khrennikov, A., Basieva, I., Pothos, E. M., and Yamato, I. (2018). Quantum probability in decision making from quantum information representation of neuronal states. Scientific Reports, 8(1):16225.

Khrennikov, A. Y. and Haven, E. (2009). Quantum mechanics and violations of the sure-thing principle: The use of probability interference and other concepts. Journal of Mathematical Psychology, 53(5):378-388.

Lewis, M. (2019). Compositional Hyponymy with Positive Operators. In Proceedings of the International Conference on Recent Advances in Natural Language Processing (RANLP 2019), pages 638-647, Varna, Bulgaria. INCOMA Ltd.

Lewis, M. (Sepember 2020). Towards logical negation for compositional distributional semantics. IfCoLog Journal of Applied Logics, 7(5):771-794.

Lorenz, R., Pearson, A., Meichanetzidis, K., Kartsaklis, D., and Coecke, B. (2021). Qnlp in practice: Running compositional models of meaning on a quantum computer. arXiv preprint arXiv:2102.12846.

Martin, A. E. and Doumas, L. A. A. (2020). Tensors and compositionality in neural systems. Philosophical Transactions of the Royal Society B: Biological Sciences, 375(1791):20190306. 
McCoy, R. T., Linzen, T., Dunbar, E., and Smolensky, P. (2019). RNNs Implicitly Implement Tensor Product Representations. In ICLR 2019 - International Conference on Learning Representations.

Meyer, F. and Lewis, M. (2020). Modelling Lexical Ambiguity with Density Matrices. In Proceedings of the 24th Conference on Computational Natural Language Learning, pages 276-290, Online. Association for Computational Linguistics.

Moore, D. W. (2002). Measuring New Types of Question-Order Effects. Public Opinion Quarterly, 66(1):8091.

Morier, D. and Bordiga, E. (1984). The Conjunction Fallacy: A Task Specific Phenomenon? Personality and Social Psychology Bulletin, 10(2).

Nielsen, M. A. and Chuang, I. L. (2010). Quantum Computation and Quantum Information: 10th Anniversary Edition. Cambridge University Press, Cambridge.

Nosofsky, R. M. (1986). Attention, similarity, and the identification-categorization relationship. Journal of Experimental Psychology: General, 115(1):39-57.

Penrose, R. (1990). The nonalgorithmic mind. Behavioral and Brain Sciences, 13(4):692-705.

Piedeleu, R., Kartsaklis, D., Coecke, B., and Sadrzadeh, M. (2015). Open System Categorical Quantum Semantics in Natural Language Processing. In $C A L C O$, page 14.

Plate, T. (1995). Holographic reduced representations. IEEE Transactions on Neural Networks, 6(3):623641.

Pothos, E. M. and Busemeyer, J. R. (2009). A quantum probability explanation for violations of 'rational' decision theory. Proceedings. Biological Sciences, 276(1665):2171-2178.

Pothos, E. M. and Busemeyer, J. R. (2013). Can quantum probability provide a new direction for cognitive modeling? Behavioral and Brain Sciences, 36(3):255-274.

Pothos, E. M., Busemeyer, J. R., and Trueblood, J. S. (2013). A quantum geometric model of similarity. Psychological Review, 120(3):679-696.

Pothos, E. M. and Trueblood, J. S. (2015). Structured representations in a quantum probability model of similarity. Journal of Mathematical Psychology, 64-65:35-43.

Rosch, E. (1975). Cognitive representations of semantic categories. Journal of Experimental Psychology: General, 104(3):192-233.

Sadrzadeh, M., Kartsaklis, D., and Balkır, E. (2018). Sentence entailment in compositional distributional semantics. Annals of Mathematics and Artificial Intelligence, 82(4):189-218.

Savage, L. J. (1954). The Foundations of Statistics. Wiley Publications in Statistics.

Smith, E. E. and Osherson, D. N. (1984). Conceptual combination with prototype concepts. Cognitive Science, 8(4):337-361.

Smolensky, P. (1990). Tensor product variable binding and the representation of symbolic structures in connectionist systems. Artificial Intelligence, 46(1-2):159-216.

Smolensky, P. and Legendre, G. (2006). The Harmonic Mind. The MIT Press.

Sozzo, S. (2014). A Quantum Probability Explanation in Fock Space for Borderline Contradictions. arXiv:1311.6050 [physics, physics:quant-ph]. 
Sozzo, S. (2015). Conjunction and negation of natural concepts: A quantum-theoretic modeling. Journal of Mathematical Psychology, 66:83-102.

Tversky, A. (1977). Features of similarity. Psychological Review, 84(4):327-352.

Tversky, A. and Kahneman, D. (1983). Extensional versus intuitive reasoning: The conjunction fallacy in probability judgment. Psychological Review, 90(4):293-315.

Tversky, A. and Shafir, E. (1992). The Disjunction Effect in Choice under Uncertainty. Psychological Science, $3(5): 305-310$.

Wang, Z. and Busemeyer, J. R. (2013). A Quantum Question Order Model Supported by Empirical Tests of an A Priori and Precise Prediction. Topics in Cognitive Science, pages n/a-n/a.

Wang, Z., Busemeyer, J. R., Atmanspacher, H., and Pothos, E. M. (2013). The Potential of Using Quantum Theory to Build Models of Cognition. Topics in Cognitive Science, pages n/a-n/a.

Widdows, D. and Peters, S. (2003). Word Vectors and Quantum Logic Experiments with negation and disjunction. Stanford University, page 14.

Wiebe, N., Bocharov, A., Smolensky, P., Troyer, M., and Svore, K. M. (2019). Quantum Language Processing. arXiv:1902.05162 [quant-ph].

Wittgenstein, L. (1953). Philosophical Investigations. Philosophische Untersuchungen. Philosophical Investigations. Philosophische Untersuchungen. Macmillan, Oxford, England. 